\title{
The roles of conic sections and elliptic curves in the global dynamics of a class of planar systems of rational difference equations
}

Sukanya Basu*

"Correspondence:

sukanyabasu@yahoo.com Department of Mathematics,

Central Michigan University, Mount Pleasant, MI 48859, USA

C2013 Basu; licensee Springer. This is an Open Access article distributed under the terms of the Creative Commons Attribution License (http://creativecommons.org/licenses/by/2.0), which permits unrestricted use, distribution, and reproduction in any medium, provided the original work is properly cited.

\section{Abstract}

Consider the class of planar systems of first-order rational difference equations

$$
\left.\begin{array}{l}
x_{n+1}=\frac{\alpha_{1}+\beta_{1} x_{n}+\gamma_{1} y_{n}}{A_{1}+B_{1} x_{n}+C_{1} y_{n}} \\
y_{n+1}=\frac{\alpha_{2}+\beta_{2} x_{n}+\gamma_{2} y_{n}}{A_{2}+B_{2} x_{n}+C_{2} y_{n}}
\end{array}\right\}, \quad n=0,1,2, \ldots,\left(x_{0}, y_{0}\right) \in \mathcal{R},
$$

where $\mathcal{R}=\left\{(x, y) \in[0, \infty)^{2}: A_{i}+B_{i} x+C_{i} y \neq 0, i=1,2\right\}$, and the parameters are nonnegative and such that both terms in the right-hand side of $\left(1^{\prime}\right)$ are nonlinear. In this paper, we prove the following discretized Poincaré-Bendixson theorem for the class of systems $\left(1^{\prime}\right)$.

If the map associated to system $\left(1^{\prime}\right)$ is bounded, then the following statements are true:

(i) If both equilibrium curves of $\left(1^{\prime}\right)$ are reducible conics, then every solution converges to one of up to four equilibria.

(ii) If exactly one equilibrium curve of $\left(1^{\prime}\right)$ is a reducible conic, then every solution converges to one of up to two equilibria.

(iii) If both equilibrium curves of $\left(1^{\prime}\right)$ are irreducible conics, then every solution converges to one of up to three equilibria or to a unique minimal period-two solution which occurs as the intersection of two elliptic curves.

In particular, system ( $\left.1^{\prime}\right)$ cannot exhibit chaos when its associated map is bounded. Moreover, we show that if both equilibrium curves of $\left(1^{\prime}\right)$ are reducible conics and the map associated to system $\left(1^{\prime}\right)$ is unbounded, then every solution converges to one of up to infinitely many equilibria or to $(0, \infty)$ or $(\infty, 0)$.

MSC: 39A05; 39A11

Keywords: difference equation; rational; global behavior; equilibrium; orbit; globally attracting; coordinatewise monotone; equilibrium curve; reducible conic; irreducible conic; minimal period-two solution

\section{Introduction and main theorem}

Consider the system of first-order rational difference equations with nonnegative parameters

$$
\left.\begin{array}{l}
x_{n+1}=\frac{\alpha_{1}+\beta_{1} x_{n}+\gamma_{1} y_{n}}{A_{1}+B_{1} x_{n}+C_{1} y_{n}} \\
y_{n+1}=\frac{\alpha_{2}+\beta_{2} x_{n}+\gamma_{2} y_{n}}{A_{2}+B_{2} x_{n}+C_{2} y_{n}}
\end{array}\right\}, \quad n=0,1,2, \ldots,\left(x_{0}, y_{0}\right) \in \mathcal{R},
$$


where $\mathcal{R}=\left\{(x, y) \in[0, \infty)^{2}: A_{i} x+B_{i} y+C_{i} \neq 0, i=1,2\right\}$, and the parameters are nonnegative and such that both terms in the right-hand side of (1) are nonlinear. The class of systems (1) has been widely studied in recent years when the RHS is both linear and nonlinear. For example, general solutions of planar linear discrete systems with constant coefficients and weak delays were studied by Diblík and Halfarová in [1] and [2]. Global behavior of solutions and basins of attraction of equilibria for special nonlinear cases of system (1) called competitive and anticompetitive systems were studied by authors such as Basu, Merino and Kulenović in [3] and [4-14]. Patterns of boundedness of nonlinear cases of system (1) were studied by Ladas et al. in [15-19]. More general results for system (1) as well its lowerand higher-dimensional counterparts were obtained by, for example, Basu and Merino in [20], by Stević, Diblík et al. in [21-24], and by Ladas et al. in [25].

The class of systems (1) was proposed in all its generality by Camouzis et al. in [26]. A number of open problems regarding (1) were also mentioned in the latter. Our goal in this paper is to give a complete qualitative description of the global behavior of solutions to all systems (1) whose maps are bounded and thus provide answers to many of the open problems in [26]. For example, we present the global dynamics of the system labeled $(14,38)$ in open problem 1 and the competitive system labeled $(15,29)$ in open problem 3 in [26]. We also give the global analysis of the following 22 systems in open problem 4 which may be competitive in some range of its parameters but nowhere cooperative: $(15, l)$ and $(30, l)$ with $l \in\{35,36,43,45,47,49\},(36,38),(36,43)$, and $(38, l),(43, l)$ with $l \in\{43,45,47,49\}$. The eight systems $(36,36),(36,45),(36,47),(36,49),(45,45),(45,47)$, $(45,49)$ and $(49,49)$ from open problem 5 , which may be competitive in a certain region of parameters, cooperative in another region of parameters and neither competitive nor cooperative in a third region of parameters, are also analyzed in this paper.

We also look at the four systems $(34,36),(34,45),(34,49)$ and $(46,49)$ from open problem 6 which may be cooperative in some range of parameters but nowhere competitive. In addition, we present the global dynamics of a number of cases of system (1) from open problem 7 which are neither competitive nor cooperative in any parameter region along with many additional cases that were not mentioned in [26], namely, cases $(k, l)$ with $k>l$. In all, we give the global dynamics of all 416 cases of nonlinear system (1) for which both members of the system are bounded along with 36 cases for which one or more members of the system are unbounded. We also show that for all of these cases, for which there exists a unique nonnegative equilibrium and no minimal period-two solutions, local stability of the equilibrium implies global attractivity. Thus we provide the answer to open problem 2.3 in [27] for the cases mentioned above.

Members of the class of systems (1) have proven to be very useful for modeling purposes in biological sciences (see [28-30]). For example, the Leslie-Gower model from theoretical ecology is the two-species competition model

$$
\left.\begin{array}{l}
x_{n+1}=\frac{b_{1} x_{n}}{1+c_{11} x_{n}+c_{12} y_{n}} \\
y_{n+1}=\frac{b_{2} y_{n}}{1+c_{21} x_{n}+c_{22} y_{n}}
\end{array}\right\}, \quad n=0,1, \ldots,\left(x_{0}, y_{0}\right) \in[0, \infty) \times[0, \infty)
$$

which can be obtained from (1) by setting $\alpha_{1}=\gamma_{1}=0$ and normalizing the other parameters. It was studied in detail by Liu and Elaydi [31], Cushing et al. [32], and Kulenović and Merino [33]. This system has the nice property that its equilibria have relatively simple algebraic formulas. Hence their local stability characters can be analyzed using stan- 
dard linearization techniques. Moreover, this system is competitive (see [34-36]). So, it is somewhat easier to analyze global behavior of its solutions.

Unfortunately, most members of class (1) do not possess either of these two nice properties of simple formulas for their equilibria and competitiveness. Another challenge faced in the study of class (1) is the presence of a large number of parameters (twelve), which makes algebraic computations involving standard linearization techniques very complicated. One also needs to analyze a large number of individual cases (2,116 cases) of (1) which is neither practical nor efficient. Finally, members of this class tend to possess multiple equilibria and minimal period-two solutions possibly at the same time. Due to these difficulties, the global dynamics of members of this class remains largely unanalyzed to date. In [3], Merino and the author introduced a new geometrical technique to analyze local and global behavior of solutions to a special case of system (E), the modified LeslieGower model

$$
\left.\begin{array}{l}
x_{n+1}=\frac{b_{1} x_{n}}{1+c_{11} x_{n}+c_{12} y_{n}}+h_{1} \\
y_{n+1}=\frac{b_{2} y_{n}}{1+c_{21} x_{n}+c_{22} y_{n}}+h_{2}
\end{array}\right\}, \quad n=0,1, \ldots,\left(x_{0}, y_{0}\right) \in[0, \infty) \times[0, \infty) .
$$

The technique is based on the analysis of slopes of equilibrium curves of the system which are defined as follows. If $T(x, y):=\left(T_{1}(x, y), T_{2}(x, y)\right)$ is a map associated to the system, then the two equilibrium curves of the system are respectively given by the formulas $T_{1}(x, y)=x$ and $T_{2}(x, y)=y$. Thus these curves are analogous to nullclines in differential equations and their intersection points are precisely the equilibria of the system. This method was then used to establish a connection between the number of equilibria of the system and their local stability. The authors were then able to use this result along with the results proved by Kulenović and Merino in [33] to give a complete qualitative description of the global dynamics of (LG-1). Also in [20], Merino and the author introduced another new method to analyze global behavior of solutions to two classes of second-order rational difference equations which are not competitive. The goal of this paper is to apply these two new techniques to analyze global behavior of solutions to the more general family of firstorder planar systems of rational difference equations (1) with nonnegative parameters. In particular, a geometrical criterion is presented to classify a large number of cases of system (E) into subclasses exhibiting similar global dynamics. Let $\mathcal{P} \subset \mathbb{R}^{12}$ be the set of nonnegative parameters $\left(\alpha_{1}, \beta_{1}, \ldots\right)$ such that the RHS terms in system (1) are nonlinear. The main theorem of this paper is as follows.

Theorem 1 If the map associated to system (1) is bounded with parameters in $\mathcal{P}$, then the following is true:

(i) If both equilibrium curves of (1) are reducible conics, that is, if

i. $C_{1}\left(C_{1} \alpha_{1}-A_{1} \gamma_{1}\right)+\gamma_{1}\left(C_{1} \beta_{1}-B_{1} \gamma_{1}\right)=0$, and

ii. $B_{2}\left(B_{2} \alpha_{2}-A_{2} \beta_{2}\right)+\beta_{2}\left(B_{2} \gamma_{2}-C_{2} \beta_{2}\right)=0$,

then system (1) has at least one and at most four equilibria. Every solution converges to an equilibrium.

(ii) If exactly one equilibrium curve of (1) is a reducible conic, that is, if either

i. $C_{1}\left(C_{1} \alpha_{1}-A_{1} \gamma_{1}\right)+\gamma_{1}\left(C_{1} \beta_{1}-B_{1} \gamma_{1}\right)=0$, or

ii. $B_{2}\left(B_{2} \alpha_{2}-A_{2} \beta_{2}\right)+\beta_{2}\left(B_{2} \gamma_{2}-C_{2} \beta_{2}\right)=0$,

then system (1) has at least one and at most two equilibria. Every solution converges to an equilibrium. 
(iii) If both equilibrium curves of (1) are irreducible conics, that is, if

i. $C_{1}\left(C_{1} \alpha_{1}-A_{1} \gamma_{1}\right)+\gamma_{1}\left(C_{1} \beta_{1}-B_{1} \gamma_{1}\right) \neq 0$, and

ii. $B_{2}\left(B_{2} \alpha_{2}-A_{2} \beta_{2}\right)+\beta_{2}\left(B_{2} \gamma_{2}-C_{2} \beta_{2}\right) \neq 0$,

then system (1) has at least one and at most three equilibria. Every solution

converges to an equilibrium or to a unique minimal period-two solution which

occurs as the intersection of two elliptic curves.

Moreover, if both equilibrium curves of (1) are reducible conics and the map associated to system (1) is unbounded, then every solution converges to one of up to infinitely many equilibria or to $(0, \infty)$ or $(\infty, 0)$.

We treat the three cases of Theorem 1 as three smaller theorems and devote three separate sections of the paper to their respective proofs. What makes case (i) of Theorem 1 relatively easy to analyze is the fact that the map $T$ associated to system (1) is coordinatewise monotone in this case. Hence the global dynamics of its orbits is relatively easy to track. In case (ii), the map $T$ is monotone in only one coordinate. Here the global dynamics of its orbits is a bit more complicated. However, the most complicated dynamics occurs in case (iii) where the map $T$ is not monotone in any coordinate. In this case, the bounded set $\mathcal{B}:=\left[\mathcal{L}_{1}, \mathcal{U}_{1}\right] \times\left[\mathcal{L}_{2}, \mathcal{U}_{2}\right]$ containing the solutions to system (1) can be subdivided into five regions of coordinatewise monotonicity based on the relative positions of a pair of vertical lines $x=K_{1}$ and $x=K_{2}$ and a pair of horizontal lines $y=L_{1}$ and $y=L_{2}$ in the set $\mathcal{B}$ as shown below:

(a) $\left\{K_{1}, K_{2}\right\} \cap\left[\mathcal{L}_{1}, \mathcal{U}_{1}\right]=\phi$ and $\left\{L_{1}, L_{2}\right\} \cap\left[\mathcal{L}_{2}, \mathcal{U}_{2}\right]=\phi$,

(b) Either $K_{2} \in\left[\mathcal{L}_{1}, \mathcal{U}_{1}\right]$ or $L_{1} \in\left[\mathcal{L}_{2}, \mathcal{U}_{2}\right]$, and $K_{1} \notin\left[\mathcal{L}_{1}, \mathcal{U}_{1}\right], L_{2} \notin\left[\mathcal{L}_{2}, \mathcal{U}_{2}\right]$,

(c) Either $K_{1} \in\left[\mathcal{L}_{1}, \mathcal{U}_{1}\right]$ or $L_{2} \in\left[\mathcal{L}_{2}, \mathcal{U}_{2}\right]$, and $K_{2} \notin\left[\mathcal{L}_{1}, \mathcal{U}_{1}\right], L_{1} \notin\left[\mathcal{L}_{2}, \mathcal{U}_{2}\right]$,

(d) $K_{2}, L_{1} \in\left[\mathcal{L}_{1}, \mathcal{U}_{1}\right]$ or $K_{1}, L_{2} \in\left[\mathcal{L}_{2}, \mathcal{U}_{2}\right]$,

(e) $K_{1}, K_{2} \in\left[\mathcal{L}_{1}, \mathcal{U}_{1}\right]$ or $L_{1}, L_{2} \in\left[\mathcal{L}_{2}, \mathcal{U}_{2}\right]$.

Here $K_{1}$ and $L_{1}$ depend on the parameter values $\alpha_{1}, \beta_{1}, \ldots, B_{1}, C_{1}$, while $K_{2}$ and $L_{2}$ depend on the parameter values $\alpha_{2}, \beta_{2}, \ldots, B_{2}, C_{2}$. To prove case (iii), we will show that there exists a nested sequence of invariant attracting boxes $\left\{\mathcal{B}_{i}\right\}_{i=1}^{\infty}$ with the property that $\mathcal{B}^{*}=\bigcap \mathcal{B}_{i}$ satisfies exactly one of the following:

(i) $\mathcal{B}^{*}=(\bar{x}, \bar{y})$.

(ii) There exist equilibria $\left(\bar{x}_{1}, \bar{y}_{1}\right) \preceq_{\text {se }}\left(\bar{x}_{2}, \bar{y}_{2}\right) \preceq_{\text {se }}\left(\bar{x}_{3}, \bar{y}_{3}\right)$ such that $\left(\bar{x}_{1}, \bar{y}_{1}\right)$ and $\left(\bar{x}_{3}, \bar{y}_{3}\right)$ lie at the north-west and south-east corners of $\mathcal{B}^{*}$, respectively, and $\left(\bar{x}_{2}, \bar{y}_{2}\right)$ lies in its interior.

(iii) There exist minimal period-two solutions $(p, q) \preceq_{\text {se }}(\bar{x}, \bar{y}) \preceq_{\text {se }}(r, s)$ such that $(p, q)$ and $(r, s)$ lie at the north-west and south-east corners of $\mathcal{B}^{*}$, respectively, and $(\bar{x}, \bar{y})$ lies in its interior.

In case (i), it is clear that the unique equilibrium $(\bar{x}, \bar{y})$ is globally attracting. In case (ii), we show that the local stability of the equilibria is determined by the slopes of the equilibrium curves at these equilibria. In case (iii), we prove that system (1) has a unique minimal period-two solution by looking at intersections of certain elliptic curves. We then use these results to give global stability results for the two cases.

This paper is organized as follows. In Section 2, we look at the admissible parameter regions and initial conditions for system (1). In Section 3, we define the notions of south-east order, competitive maps and equilibrium curves of system (1). In Section 4, we look at explicit formulas for the cases of system (1) for which the associated map $T(x, y)$ is bounded. 
In Section 5, we look at regions of coordinatewise monotonicity for the map $T(x, y)$. Sections 6 and 7 respectively deal with the case where both equilibrium curves of system (1) are reducible conics and the case where exactly one of them is a reducible conic. Sections 8.1-8.4 respectively deal with the number of nonnegative equilibria, local stability of equilibria, existence and uniqueness of minimal period-two solutions, and global behavior of solutions of system (1) when both equilibrium curves are irreducible conics.

\section{Parameter regions and initial conditions}

In this section, we look at conditions that the parameters $\alpha_{1}, \beta_{1}, \ldots, B_{2}$ and $C_{2}$ of system (1) must satisfy in order to be included in the set $\mathcal{P}$ introduced in Theorem 1 in the previous section. In particular, note that the parameters in $\mathcal{P}$ must satisfy the following inequalities:

$$
\left.\begin{array}{l}
B_{i}+C_{i}>0 \\
\alpha_{i}+\beta_{i}+\gamma_{i}>0 \\
A_{i}+B_{i}+C_{i}>0 \\
\alpha_{i}+\beta_{i}+A_{i}+B_{i}>0 \\
\alpha_{i}+\gamma_{i}+A_{i}+C_{i}>0 \\
\beta_{i}+\gamma_{i}+B_{i}+C_{i}>0
\end{array}\right\}, \quad i=1,2 .
$$

The reasons for these inequalities are as follows. If $B_{i}=C_{i}=0$ for $i \in\{1,2\}$, then at least one of the members of system (1) becomes linear. Since we are interested in studying nonlinear rational systems of difference equations belonging to class (1), we will ignore these cases. Next, note that if $\alpha_{i}+\beta_{i}+\gamma_{i}=0$ for $i=1$ or 2 , then at least one of the members of system (1) becomes trivial causing the latter to reduce to a difference equation. Since we are interested in studying systems of difference equations belonging to class (1), we will ignore these cases as well. Similarly, if $A_{i}=B_{i}=\alpha_{i}=\beta_{i}=0$ or $A_{i}=C_{i}=\alpha_{i}=\gamma_{i}=0$ for $i \in\{1,2\}$, then at least one of the members of system (1) becomes constant, and we have the same situation as before, which we want to avoid.

The assumption that each of the twelve parameters $\alpha_{i}, \beta_{i}, \gamma_{i}, A_{i}, B_{i}$ and $C_{i}$ for $i \in\{1,2\}$ can be zero or positive and the inequalities in hypotheses (2) imply that for $i=1$ there are $2^{3}-1=7$ ways to choose the numerator of the first member of system (1) excluding the trivial case $\alpha_{1}=\beta_{1}=\gamma_{1}=0$. Similarly, there are seven ways to choose the denominator. Thus there are $7 \times 7=49$ ways to choose the first member of system (1). Out of these, only $49-3=46$ choices satisfy the last two inequalities in hypotheses (2). Similarly, there are 46 choices for the second member of system (1). In all, there are $46 \times 46=2,116$ ways to choose systems belonging to class (1). Moreover, the initial condition $\left(x_{0}, y_{0}\right) \in \mathcal{R}$ must be chosen according to Table 1 in order to avoid division by zero.

Table 1 Regions $\mathcal{R}$ of initial conditions

\begin{tabular}{ll}
\hline Parameter condition & $\mathcal{R}$ \\
\hline$A_{1}>0, A_{2}>0$ & {$[0, \infty) \times[0, \infty)$} \\
$\left(A_{1}=B_{1}=0, A_{2} \neq 0\right)$ or $\left(A_{2}=B_{2}=0, A_{1} \neq 0\right)$ & {$[0, \infty) \times(0, \infty)$} \\
$\left(A_{1}=C_{1}=0, A_{2} \neq 0\right)$ or $\left(A_{2}=C_{2}=0, A_{1} \neq 0\right)$ & $(0, \infty) \times[0, \infty)$ \\
$A_{1}=B_{1}=0, A_{2}=B_{2}=0$ & {$[0, \infty) \times(0, \infty)$} \\
$A_{1}=C_{1}=0, A_{2}=C_{2}=0$ & $(0, \infty) \times[0, \infty)$ \\
$A_{1}=C_{1}=0, A_{2}=B_{2}=0$ & {$[0, \infty) \times[0, \infty) \backslash(0,0)$} \\
$A_{1}=B_{1}=0, A_{2}=C_{2}=0$ & {$[0, \infty) \times[0, \infty)$} \\
$\left(A_{1}=0, B_{1} \neq 0, C_{1} \neq 0\right)$ or $\left(A_{2}=0, B_{2} \neq 0, C_{2} \neq 0\right)$ & {$[0, \infty) \times[0, \infty) \backslash(0,0)$} \\
\hline
\end{tabular}




\section{Important definitions}

In this section, we provide some key definitions which we will frequently refer to throughout this paper. Let $T$ be the map associated with system (1), that is,

$$
\begin{aligned}
T(x, y) & :=\left(\frac{\alpha_{1}+\beta_{1} x+\gamma_{1} y}{A_{1}+B_{1} x+C_{1} y}, \frac{\alpha_{2}+\beta_{2} x+\gamma_{2} y}{A_{2}+B_{2} x+C_{2} y}\right) \\
& :=\left(T_{1}(x, y), T_{2}(x, y)\right) .
\end{aligned}
$$

Let $T_{1}$ and $T_{2}$ be the coordinate functions of $T$, that is,

$$
T_{1}(x, y)=\frac{\alpha_{1}+\beta_{1} x+\gamma_{1} y}{A_{1}+B_{1} x+C_{1} y} \quad \text { and } \quad T_{2}(x, y)=\frac{\alpha_{2}+\beta_{2} x+\gamma_{2} y}{A_{2}+B_{2} x+C_{2} y} .
$$

Then system (1) can be written as

$$
\left(\begin{array}{l}
x_{n+1} \\
y_{n+1}
\end{array}\right)=\left(\begin{array}{l}
T_{1}\left(x_{n}, y_{n}\right) \\
T_{2}\left(x_{n}, y_{n}\right)
\end{array}\right)=T\left(\begin{array}{l}
x_{n} \\
y_{n}
\end{array}\right) .
$$

Definition 1 For a given choice of parameters in $\mathcal{P}$, we say that system (1) is bounded if the associated map $T$ is bounded, i.e., if there exist nonnegative constants $c_{1}, C_{1}, c_{2}$ and $C_{2}$ such that

$$
\begin{aligned}
& c_{1} \leq T_{1}(x, y) \leq C_{1}, \\
& c_{2} \leq T_{2}(x, y) \leq C_{2} .
\end{aligned}
$$

Definition 2 The south-east order $\preceq_{\text {se }}$ on $\mathbb{R}^{2}$ is defined as follows:

$$
\left(x_{1}, y_{1}\right) \preceq_{\text {se }}\left(x_{2}, y_{2}\right) \quad \Longleftrightarrow \quad x_{1}<x_{2} \quad \text { and } \quad y_{1}>y_{2} \text {. }
$$

Definition 3 A continuous map $T: \mathbb{R}^{2} \rightarrow \mathbb{R}^{2}$ is said to be competitive if it is monotone with respect to the south-east ordering $\preceq_{\text {se }}$.

Remark One can easily check that the Jacobian of a competitive map satisfies the sign structure $\left(\begin{array}{c}+- \\ -+\end{array}\right)$.

Definition 4 The equilibrium curves $E_{1}$ and $E_{2}$ of system (1) are the sets

$$
E_{1}:=\left\{(x, y) \in \mathbb{R}^{2}: x=T_{1}(x, y)\right\}, \quad E_{2}:=\left\{(x, y) \in \mathbb{R}^{2}: y=T_{2}(x, y)\right\} .
$$

Note that $E_{1}$ and $E_{2}$ are loci of conic sections:

$$
\begin{aligned}
& E_{1}: B_{1} x^{2}+C_{1} x y+\left(A_{1}-\beta_{1}\right) x-\gamma_{1} y-\alpha_{1}=0, \\
& E_{2}: C_{2} y^{2}+B_{2} x y+\left(A_{2}-\gamma_{2}\right) y-\beta_{2} x-\alpha_{2}=0 .
\end{aligned}
$$

It follows from analytic geometry that if the discriminants of $E_{1}$ and $E_{2}$ are respectively nonzero, that is, if the parameters of $E_{1}$ and $E_{2}$ respectively satisfy the following two conditions, then the equilibrium curves $E_{1}$ and $E_{2}$ must respectively be irreducible conics (parabolas, hyperbolas or ellipses): 
i. $C_{1}\left(C_{1} \alpha_{1}-A_{1} \gamma_{1}\right)+\gamma_{1}\left(C_{1} \beta_{1}-B_{1} \gamma_{1}\right) \neq 0$,

ii. $B_{2}\left(B_{2} \alpha_{2}-A_{2} \beta_{2}\right)+\beta_{2}\left(B_{2} \gamma_{2}-C_{2} \beta_{2}\right) \neq 0$.

Moreover, since $C_{1} \geq 0$ and $B_{2} \geq 0, E_{1}$ and $E_{2}$ cannot be ellipses. In this paper, we consider three separate cases, namely, the cases where (i) both $E_{1}$ and $E_{2}$ are reducible conics, (ii) exactly one of $E_{1}$ and $E_{2}$ is a reducible conic, and (iii) both $E_{1}$ and $E_{2}$ are irreducible conics.

\section{Bounded cases of system (1)}

In this section, we look at bounded cases of system (1), that is, special cases of system (1) for which all solutions with nonnegative/positive initial conditions are bounded. These cases have the property that their associated maps are bounded. They are obtained by setting one or more of the twelve nonnegative parameters $\alpha_{1}, \beta_{1}, \gamma_{1}, A_{1}, B_{1}, C_{1}, \alpha_{2}, \beta_{2}, \gamma_{2}$, $A_{2}, B_{2}$ and $C_{2}$ to zero in system (1) and have been studied in great detail by Ladas et al. in, for example, [27, 37] and [38], to name a few. For a more complete list of important work done in analyzing the boundedness of a large number of special cases of system (1) by Ladas et al., the reader is referred to references [4-19, 25, 39-42]. Such systems have been referred to as having boundedness characterization (B, B) in these papers. In particular, explicit formulas for many of these systems were given in Appendices 1 and 2 of reference [37].

In this section, we show that there are at least 564 bounded nonlinear cases of system (1). We also give explicit formulas for all of these 564 cases. This result is important because it shows that there are enough bounded nonlinear cases of system (1) (at least 564 cases!) to warrant the study conducted in this paper. It is stated next. Denote the expressions on the RHS of system (1) by $T_{1}\left(x_{n}, y_{n}\right)$ and $T_{2}\left(x_{n}, y_{n}\right)$ respectively as shown below:

$$
\begin{aligned}
& x_{n+1}=T_{1}\left(x_{n}, y_{n}\right), \\
& y_{n+1}=T_{2}\left(x_{n}, y_{n}\right) .
\end{aligned}
$$

Theorem 2 If the functions $T_{1}\left(x_{n}, y_{n}\right)$ and $T_{2}\left(x_{n}, y_{n}\right)$ in the RHS of (5) have one of the formulas given below, then system (1) is bounded:

(a) $T_{1}\left(x_{n}, y_{n}\right)$ and $T_{2}\left(x_{n}, y_{n}\right)$ are given by one of the formulas in the right-hand column of Table 2.

(b) $T_{1}\left(x_{n}, y_{n}\right)$ is given by one of the formulas in the right-hand column of Table 2 and $T_{2}\left(x_{n}, y_{n}\right)$ is given by one of the following formulas:

$$
\begin{aligned}
& \frac{\beta_{2} x_{n}}{A_{2}}, \quad \frac{\alpha_{2}+\beta_{2} x_{n}}{A_{2}}, \quad \frac{\beta_{2} x_{n}}{A_{2}+C_{2} y_{n}}, \frac{\alpha_{2}+\beta_{2} x_{n}}{A_{2}+C_{2} y_{n}}, \\
& \frac{\beta_{2} x_{n}+\gamma_{2} y_{n}}{A_{2}+C_{2} y_{n}}, \quad \frac{\alpha_{2}+\beta_{2} x_{n}+\gamma_{2} y_{n}}{A_{2}+C_{2} y_{n}} .
\end{aligned}
$$

(c) $T_{2}\left(x_{n}, y_{n}\right)$ is given by one of the formulas in the right-hand column of Table 2 and $T_{1}\left(x_{n}, y_{n}\right)$ is given by one of the following formulas:

$$
\begin{array}{lll}
\frac{\gamma_{1} y_{n}}{A_{1}}, \quad \frac{\alpha_{1}+\gamma_{1} y_{n}}{A_{1}}, \quad \frac{\gamma_{1} y_{n}}{A_{1}+B_{1} x_{n}}, & \frac{\alpha_{1}+\gamma_{1} y_{n}}{A_{1}+B_{1} x_{n}}, \\
\frac{\beta_{1} x_{n}+\gamma_{1} y_{n}}{A_{1}+B_{1} x_{n}}, & \frac{\alpha_{1}+\beta_{1} x_{n}+\gamma_{1} y_{n}}{A_{1}+B_{1} x_{n}} . &
\end{array}
$$


Table 2 Some formulas for $T_{1}\left(x_{n}, y_{n}\right)$ and $T_{2}\left(x_{n}, y_{n}\right)$ for which system (1) is bounded

\begin{tabular}{|c|c|c|c|}
\hline & $\begin{array}{l}\text { Number of terms in the } \\
\text { denominator of } T_{i}\left(x_{n}, y_{n}\right), \\
i=1,2\end{array}$ & $\begin{array}{l}\text { Formula for the } \\
\text { denominator of } T_{i}\left(x_{n}, y_{n}\right), \\
i=1,2\end{array}$ & $\begin{array}{l}\text { Formula for } T_{i}\left(x_{n}, y_{n}\right), i=1,2, \text { for } \\
\text { which system }(1) \text { is bounded }\end{array}$ \\
\hline 1. & Three & $A_{i}+B_{i} x_{n}+C_{i} y_{n}$ & $\begin{array}{l}\frac{\alpha_{i}}{A_{i}+B_{i} x_{n}+C_{i} y_{n}}, \frac{\beta_{i} x_{n}}{A_{i}+B_{i} x_{n}+C_{i} y_{n}}, \frac{\gamma_{i} y_{n}}{A_{i}+B_{i} x_{n}+C_{i} y_{n}}, \\
\frac{\alpha_{i}+\beta_{i} x_{n}}{A_{i}+B_{i} x_{n}+C_{i} y_{n}}, \frac{\alpha_{i}+\gamma_{i} y_{n}}{A_{i}+B_{i} x_{n}+C_{i} y_{n}}, \frac{\beta_{i} x_{n}+\gamma_{i} y_{n}}{A_{i}+B_{i} x_{n}+C_{i} y_{n}}, \\
\frac{\alpha_{i}+\beta_{i} x_{n}+\gamma_{i} y_{n}}{A_{i}+B_{i} x_{n}+C_{i} y_{n}}\end{array}$ \\
\hline 2. & Two & $\begin{array}{l}B_{i} x_{n}+C_{i} y_{n} \\
A_{i}+B_{i} x_{n} \\
A_{i}+C_{i} y_{n}\end{array}$ & $\begin{array}{l}\frac{\beta_{i} x_{n}+\gamma_{i} y_{n}}{B_{i} x_{n}+C_{i} y_{n}}, \frac{\beta_{i} x_{n}}{B_{i} x_{n}+C_{i} y_{n}}, \frac{\gamma_{i} y_{n}}{B_{i} x_{n}+C_{i} y_{n}} \\
\frac{\alpha_{i}}{A_{i}+B_{i} x_{n}}, \frac{\beta_{i} x_{n}}{A_{i}+B_{i} x_{n}}, \frac{\alpha_{i}+\beta_{i} x_{n}}{A_{i}+B_{i} x_{n}} \\
\frac{\alpha_{i}}{A_{i}+C_{i} y_{n}}, \frac{\gamma_{i} y_{n}}{A_{i}+C_{i} y_{n}}, \frac{\alpha_{i}+\gamma_{i} y_{n}}{A_{i}+C_{i} y_{n}}\end{array}$ \\
\hline 3. & One & $\begin{array}{l}A_{i} \\
B_{i} x_{n} \\
C_{i} y_{n}\end{array}$ & $\begin{array}{l}\alpha_{i} / A_{i} \\
\beta_{i} / B_{i} \\
\gamma_{i} / C_{i}\end{array}$ \\
\hline
\end{tabular}

Thus there are at least 589 bounded cases of system (1) of which 564 cases are nonlinear.

Proof To see the proof of part (a) of the theorem, observe that if $T_{1}\left(x_{n}, y_{n}\right)$ has the first formula in the RHS of Table 2 case 1 with $i=1$, then one can respectively choose lower and upper bounds $\mathcal{L}_{1}$ and $\mathcal{U}_{1}$ for $T_{1}\left(x_{n}, y_{n}\right)$ as follows:

$$
\mathcal{L}_{1}:=0<\frac{\alpha_{1}}{A_{1}+B_{1} x_{n}+C_{1} y_{n}}<\frac{\alpha_{1}\left(1+x_{n}+y_{n}\right)}{\min \left\{A_{1}, B_{1}, C_{1}\right\}\left(1+x_{n}+y_{n}\right)}=\frac{\alpha_{1}}{\min \left\{A_{1}, B_{1}, C_{1}\right\}}=: \mathcal{U}_{1} .
$$

This idea extends to the other formulas in case 1 as well. For the last formula in case 1 , one can do even better with the choice of bounds as shown below:

$$
\mathcal{L}_{1}:=\frac{\min \left\{\alpha_{1}, \beta_{1}, \gamma_{1}\right\}}{\max \left\{A_{1}, B_{1}, C_{1}\right\}}<\frac{\alpha_{1}+\beta_{1} x_{n}+\gamma_{1} y_{n}}{A_{1}+B_{1} x_{n}+C_{1} y_{n}}<\frac{\max \left\{\alpha_{1}, \beta_{1}, \gamma_{1}\right\}}{\min \left\{A_{1}, B_{1}, C_{1}\right\}}=: \mathcal{U}_{1}
$$

A similar idea can be used to find bounds for the formulas in case 2 of Table 1. In case 3, the bounds are trivial since the formulas are constant to begin with. Moreover, if $T_{2}\left(x_{n}, y_{n}\right)$ has one of the formulas in Table 2 with $i=2$, then one can find lower and upper bounds $\mathcal{L}_{2}$ and $\mathcal{U}_{2}$ for it in the same manner as before. In addition, if $T_{2}\left(x_{n}, y_{n}\right)$ has the first formula in (6), then $\mathcal{L}_{2}$ and $\mathcal{U}_{2}$ can be chosen as follows:

$$
\mathcal{L}_{2}:=\frac{\beta_{2} \mathcal{L}_{1}}{A_{2}}<\frac{\beta_{2} x_{n}}{A_{2}}<\frac{\beta_{2} \mathcal{U}_{1}}{A_{2}}=: \mathcal{U}_{2} .
$$

One can similarly find $\mathcal{L}_{2}$ and $\mathcal{U}_{2}$ for the second case in (6). In the third case, one can choose

$$
\mathcal{L}_{2}:=0<\frac{\beta_{2} x_{n}}{A_{2}+C_{2} y_{n}}<\frac{\beta_{2} \mathcal{U}_{1}}{A_{2}}=: \mathcal{U}_{2} .
$$

The fourth case in (6) is similar. In the fifth case, one can choose

$$
\begin{aligned}
\mathcal{L}_{2} & :=\frac{\min \left\{\beta_{2} \mathcal{L}_{1}, \gamma_{2}\right\}}{\max \left\{A_{2}, C_{2}\right\}}<\frac{\beta_{2} \mathcal{L}_{1}+\gamma_{2} y_{n}}{A_{2}+C_{2} y_{n}}<\frac{\beta_{2} x_{n}+\gamma_{2} y_{n}}{A_{2}+C_{2} y_{n}} \\
& <\frac{\beta_{2} \mathcal{U}_{1}+\gamma_{2} y_{n}}{A_{2}+C_{2} y_{n}}<\frac{\max \left\{\beta_{2} \mathcal{U}_{1}, \gamma_{2}\right\}}{\min \left\{A_{2}, C_{2}\right\}}=: \mathcal{U}_{2} .
\end{aligned}
$$


The bounds for the last case in (6) can be found in a similar manner. The formulas in (7) are almost identical to the formulas in (6) with $A_{2}, \beta_{2}$ and $x_{n}$ respectively replaced by $A_{1}, \gamma_{1}$ and $y_{n}$. Hence their lower and upper bounds $\mathcal{L}_{2}$ and $\mathcal{U}_{2}$ can be found in a similar fashion as in (6). It follows from the previous discussion that there are $7+3+3+3+3=19$ bounded formulas for $T_{1}\left(x_{n}, y_{n}\right)$ and another 19 bounded formulas for $T_{2}\left(x_{n}, y_{n}\right)$ in cases (i)-(iv) of Table 2 of part (a). In all, there are $19 \times 19=361$ bounded cases of system 1 in part (a) and $19 \times 6=114$ bounded cases each in parts (b) and (c). This gives a total of $361+2(114)=589$ bounded cases of system 1 from parts (a), (b) and (c). Moreover, there are $3 \times 3=9$ ways to pair $T_{1}\left(x_{n}, y_{n}\right)$ and $T_{2}\left(x_{n}, y_{n}\right)$ so that both of them are constant in the RHS of (5): three choices for $T_{1}\left(x_{n}, y_{n}\right)$ from Table 2 case 3 when $i=1$ combined with three choices for $T_{2}\left(x_{n}, y_{n}\right)$ from Table 2 case 3 when $i=2$. In addition, the first two formulas in both parts (b) and (c) of the theorem are linear. They can be combined to give $2 \times 2=4$ cases where $T_{1}\left(x_{n}, y_{n}\right)$ and $T_{2}\left(x_{n}, y_{n}\right)$ are both linear in the RHS of (5). Finally, there are $2 \times 3=6$ ways each to respectively combine the two linear formulas in parts (b) and (c) with those in Table 2 case 3 so that the RHS of (5) is a combination of a linear formula and a constant formula. This gives a total of $6+6=12$ cases. To conclude, there are $9+4+12=25$ linear or constant cases out of the 589 bounded cases we originally identified above, which leaves us with $589-25=564$ bounded nonlinear cases of system (1).

The goal of this paper is to give a complete qualitative description of the global behavior of solutions to all bounded nonlinear cases of system (1) including the 564 bounded nonlinear cases mentioned in Theorem 2 above.

\section{Regions of coordinatewise monotonicity for the map $T$}

When both equilibrium curves are irreducible conics, the map $T(x, y)$ associated to bounded system (1) is not coordinatewise monotone throughout its bounded domain of definition. In this subsection, we will identify regions of coordinatewise monotonicity of the map $T(x, y)$. These regions will play a crucial role in determining the global behavior of solutions to system (1) when both equilibrium curves are irreducible conics.

Lemma 1 The following statements are true:

(i) If $B_{1} \gamma_{1}-C_{1} \beta_{1}=0$, then the partial derivatives of the functions $T_{1}(x, y)$ are continuous on $(0, \infty)^{2}$ and have constant sign on the set $\mathcal{B}$.

(ii) If $B_{2} \gamma_{2}-C_{2} \beta_{2}=0$, then the partial derivatives of the functions $T_{2}(x, y)$ are continuous on $(0, \infty)^{2}$ and have constant sign on the set $\mathcal{B}$.

Proof We give the proof of part (i). The proof of part (ii) is similar and we skip it. Note that by hypotheses (2), $B_{1}+C_{1}>0$. First, suppose $B_{1} \neq 0$ and $C_{1} \neq 0$. Solving for $\gamma_{1}$ in $B_{1} \gamma_{1}-C_{1} \beta_{1}=0$ and substituting in $\frac{\partial}{\partial x} T_{1}(x, y)$ and $\frac{\partial}{\partial y} T_{1}(x, y)$, we get that $\frac{\partial}{\partial x} T_{1}(x, y)=$ $-\frac{B_{1} \alpha_{1}-A_{1} \beta_{1}}{\left(A_{1}+B_{1} x+C_{1} y\right)^{2}}$ and $\frac{\partial}{\partial y} T_{1}(x, y)=-\frac{C_{1}\left(B_{1} \alpha_{1}-A_{1} \beta_{1}\right)}{B_{1}\left(A_{1}+B_{1} x+C_{1} y\right)^{2}}$. When $B_{1}=0$ and $C_{1} \neq 0$, the hypothesis implies that $\beta_{1}=0$. In this case, $D_{1} T_{1}(x, y)=0$ and $D_{2} T_{1}(x, y)=-\frac{C_{1}\left(B_{1} \alpha_{1}-A_{1} \beta_{1}\right)}{B_{1}\left(A_{1}+C_{1} y\right)^{2}}$. Finally, when $B_{1} \neq 0$ and $C_{1}=0$, one must have $\gamma_{1}=0$ and hence $D_{1} T_{1}(x, y)=-\frac{B_{1} \alpha_{1}-A_{1} \beta_{1}}{\left(A_{1}+B_{1} x\right)^{2}}$ and $D_{2} T_{1}(x, y)=0$. Clearly, in all three cases the partial derivatives of $T_{1}(x, y)$ have constant sign on the set $\mathcal{B}$.

We will need the following elementary result, which is given here without a proof. 
Lemma 2 Suppose $B_{i} \gamma_{i}-C_{i} \beta_{i} \neq 0$ for $i=1,2$. The functions $T_{i}(x, y), i=1,2$, have continuous partial derivatives on $(0, \infty)^{2}$, and

i. $D_{1} T_{i}(x, y)=0$ if and only if $y=-\frac{B_{i} \alpha_{i}-A_{i} \beta_{i}}{B_{i} \gamma_{i}-C_{i} \beta_{i}}$, and $D_{1} T_{i}(x, y)>0$ if and only if $\left(C_{i} \beta_{i}-B_{i} \gamma_{i}\right) y>B_{i} \alpha_{i}-A_{i} \beta_{i}$.

ii. $D_{2} T_{i}(x, y)=0$ if and only if $x=\frac{C_{i} \alpha_{i}-A_{i} \gamma_{i}}{B_{i} \gamma_{i}-C_{i} \beta_{i}}$, and $D_{2} T_{i}(x, y)>0$ if and only if

$\left(B_{i} \gamma_{i}-C_{i} \beta_{i}\right) x>C_{i} \alpha_{i}-A_{i} \gamma_{i}$.

For the rest of this paper, we will need to refer to the relative positions of $K_{i}$ and $L_{i}$ where the partial derivatives of $T_{i}(x, y)$ change sign for $i=1,2$. The explicit formulas for $K_{i}$ and $L_{i}$ for $i=1,2$ are given in the following definition. Their relative positions according to different parameter regions are shown in the Appendix for convenience.

Definition 5 If $B_{1} \gamma_{1}-C_{1} \beta_{1} \neq 0$ and $B_{2} \gamma_{2}-C_{2} \beta_{2} \neq 0$, set

$$
\begin{array}{ll}
K_{1}:=\frac{C_{1} \alpha_{1}-A_{1} \gamma_{1}}{B_{1} \gamma_{1}-C_{1} \beta_{1}}, & L_{1}:=-\frac{B_{1} \alpha_{1}-A_{1} \beta_{1}}{B_{1} \gamma_{1}-C_{1} \beta_{1}}, \\
K_{2}:=\frac{C_{2} \alpha_{2}-A_{2} \gamma_{2}}{B_{2} \gamma_{2}-C_{2} \beta_{2}}, & L_{2}:=-\frac{B_{2} \alpha_{2}-A_{2} \beta_{2}}{B_{2} \gamma_{2}-C_{2} \beta_{2}} .
\end{array}
$$

Lemma 3 The following statements are true:

(i) $K_{1} \in[0, \infty)^{2}$ if and only if $L_{1} \notin[0, \infty)^{2}$;

(ii) $K_{2} \in[0, \infty)^{2}$ if and only if $L_{2} \notin[0, \infty)^{2}$.

Proof We give the proof of part (i). The proof of part (ii) is similar and we skip it. Suppose $K_{1} \in[0, \infty)^{2}$ and $L_{1} \in[0, \infty)^{2}$. Then the parameters $\alpha_{1}, \beta_{1}, \gamma_{1}, A_{1}, B_{1}, C_{1}, \alpha_{2}, \beta_{2}, \gamma_{2}, A_{2}$, $B_{2}$ and $C_{2}$ must satisfy one of the following:

(a) $B_{1} \gamma_{1}-C_{1} \beta_{1}>0, B_{1} \alpha_{1}-A_{1} \beta_{1}<0, C_{1} \alpha_{1}-A_{1} \gamma_{1} \geq 0$;

(b) $B_{1} \gamma_{1}-C_{1} \beta_{1}<0, B_{1} \alpha_{1}-A_{1} \beta_{1} \geq 0, C_{1} \alpha_{1}-A_{1} \gamma_{1}<0$.

Note that $A_{1}, B_{1}$ and $C_{1}$ must be strictly positive in this case in order to avoid contradicting the inequalities in (a) and (b). Hence one can respectively rewrite the inequalities in (a) and (b) as

$$
\frac{\alpha_{1}}{A_{1}} \geq \frac{\gamma_{1}}{C_{1}}>\frac{\beta_{1}}{B_{1}}>\frac{\alpha_{1}}{A_{1}} \quad \text { and } \quad \frac{\alpha_{1}}{A_{1}} \geq \frac{\beta_{1}}{B_{1}}>\frac{\gamma_{1}}{C_{1}}>\frac{\alpha_{1}}{A_{1}},
$$

giving a contradiction.

\section{When both $E_{1}$ and $E_{2}$ are reducible conics}

In this section, we discuss global behavior of solutions when both equilibrium curves $E_{1}$ and $E_{2}$ are reducible conics, that is, both $E_{1}$ and $E_{2}$ are pairs of parallel, perpendicular or transversal (non-perpendicular) lines. In order for this to be true, both $E_{1}$ and $E_{2}$ must have one of the forms given below:

$$
\begin{aligned}
& E_{1}: \begin{cases}\text { (a) } B_{1} x^{2}+\left(A_{1}-\beta_{1}\right) x-\alpha_{1}=0, & \text { where } B_{1}>0, C_{1}=\gamma_{1}=0, \\
\text { (b) } x\left(C_{1} y+A_{1}-\beta_{1}\right)=0, & \text { where } C_{1}>0, \alpha_{1}=\gamma_{1}=0, B_{1}=0, \\
\text { (c) } x\left(B_{1} x+C_{1} y+A_{1}-\beta_{1}\right)=0, & \text { where } C_{1}>0, \alpha_{1}=\gamma_{1}=0, B_{1}>0,\end{cases} \\
& E_{2}:\left\{\begin{array}{lll}
\text { (a) } C_{2} y^{2}+\left(A_{2}-\gamma_{2}\right) y-\alpha_{2}=0, & \text { where } C_{2}>0, B_{2}=\beta_{2}=0, \\
\text { (b) } y\left(B_{2} x+A_{2}-\gamma_{2}\right)=0, & \text { where } B_{2}>0, \alpha_{2}=\beta_{2}=0, C_{2}=0, \\
\text { (c) } y\left(C_{2} y+B_{2} x+A_{2}-\gamma_{2}\right)=0, & \text { where } B_{2}>0, \alpha_{2}=\beta_{2}=0, C_{2}>0 .
\end{array}\right.
\end{aligned}
$$


Remark The missing parameters in the equations in (8) are assumed to be nonnegative. Also note that:

(i) In cases (a), $E_{1}$ and $E_{2}$ each belong to a pair of parallel lines. The corresponding members of system (1) have the forms

$$
\left.x_{n+1}=\frac{\alpha_{1}+\beta_{1} x_{n}}{A_{1}+B_{1} x_{n}}, \quad y_{n+1}=\frac{\alpha_{2}+\gamma_{2} y_{n}}{A_{2}+C_{2} y_{n}}, \quad \text { where } \begin{array}{l}
C_{1}=\gamma_{1}=0 \\
B_{2}=\beta_{2}=0
\end{array}\right\} .
$$

(ii) In cases (b), $E_{1}$ and $E_{2}$ each belong to a pair of perpendicular lines. The corresponding members of system (1) look like

$$
\left.x_{n+1}=\frac{\beta_{1} x_{n}}{A_{1}+C_{1} y_{n}}, \quad y_{n+1}=\frac{\gamma_{2} y_{n}}{A_{2}+B_{2} x_{n}}, \quad \text { where } \begin{array}{l}
C_{1}>0, \alpha_{1}=\gamma_{1}=0, B_{1}=0 \\
B_{2}>0, \alpha_{2}=\beta_{2}=0, C_{2}=0
\end{array}\right\} .
$$

(iii) In cases (c), $E_{1}$ and $E_{2}$ belong to a pair of non-perpendicular transversal lines each. The corresponding members of system (1) have the forms

$$
\begin{aligned}
& x_{n+1}=\frac{\beta_{1} x_{n}}{A_{1}+B_{1} x_{n}+C_{1} y_{n}}, \quad y_{n+1}=\frac{\gamma_{2} y_{n}}{A_{2}+B_{2} x_{n}+C_{2} y_{n}}, \\
& \text { where } \left.\begin{array}{c}
C_{1}>0, \alpha_{1}=\gamma_{1}=0, B_{1}>0 \\
B_{2}>0, \alpha_{2}=\beta_{2}=0, C_{2}>0
\end{array}\right\} .
\end{aligned}
$$

Note that the first equation in (i) involving $x_{n+1}$ actually consists of six separate equations corresponding to three cases each for $A_{i} \neq 0$ and $A_{i}=0$. These three cases are: (a) $\alpha_{1}=0$, $\beta_{1} \neq 0$, (b) $\alpha_{1} \neq 0, \beta_{1}=0$ and (c) $\alpha_{1} \neq 0, \beta_{1} \neq 0$. The same is true for the second equation in (i) involving $y_{n+1}$. Similarly, the two equations in (ii) each consist of two separate equations, namely, the one with $A_{i} \neq 0$ and the one with $A_{i}=0$ for $i=1,2$. The same is true of (iii).

Thus this section establishes global behavior of solutions of system (1) when its members are combinations of any of the $6+2+2=10$ forms for $x_{n+1}$ with any of the ten forms for $y_{n+1}$ given in (i)-(iii) of the last remark. This gives rise to 100 explicit planar systems of firstorder rational difference equations with positive parameters. It is a direct consequence of Table 2 in Theorem 2 that the equations in (i) and (iii) are bounded while the equations in (ii) are unbounded. Thus there are a total of $(6+2) \times(6+2)=64$ bounded systems out of the 100 systems. Moreover, if both members of (1) have the forms given in (iii) and, in addition, $A_{1}>0$ and $A_{2}>0$, then the resulting system is the well-known Leslie-Gower model from theoretical ecology whose global dynamics was analyzed by Cushing et al. in [32]. The main theorem of this section is the following.

Theorem 3 If system (1) is bounded and if both its equilibrium curves $E_{1}$ and $E_{2}$ are reducible conics, that is, if

i. $C_{1}\left(C_{1} \alpha_{1}-A_{1} \gamma_{1}\right)+\gamma_{1}\left(C_{1} \beta_{1}-B_{1} \gamma_{1}\right)=0$, and

ii. $B_{2}\left(B_{2} \alpha_{2}-A_{2} \beta_{2}\right)+\beta_{2}\left(B_{2} \gamma_{2}-C_{2} \beta_{2}\right)=0$,

then it has at least one and at most four equilibria. Every solution converges to an equilibrium.

We discuss the proof of Theorem 3 in Section 6.2. But first we establish the number of nonnegative equilibria of system (1) when both its equilibrium curves are reducible conics. 


\subsection{Number of nonnegative equilibria}

The main theorem of this subsection is the following.

Theorem 4 If system (1) is bounded and satisfies the hypotheses of Theorem 3, then it has at least one and at most four equilibria in $[0, \infty)^{2}$. Moreover,

(a) If there exists one equilibrium, then it must be $(0,0)$ or an interior equilibrium.

(b) If there exist two equilibria, then they must include an axis equilibrium.

(c) If there exist three equilibria, then they must consist of $(0,0)$ and an equilibrium on each axis.

(d) If there exist four equilibria, then they must consist of $(0,0)$, an equilibrium on each axis and an interior equilibrium.

Proof It follows from the discussion preceding this subsection that $E_{1}$ must have one of the following forms:

(a) $B_{1} x^{2}+\left(A_{1}-\beta_{1}\right) x-\alpha_{1}=0$, where $C_{1}=\gamma_{1}=0$,

(b) $x\left(C_{1} y+A_{1}-\beta_{1}\right)=0$, where $C_{1}>0, \alpha_{1}=\gamma_{1}=0, B_{1}=0$,

(c) $x\left(B_{1} x+C_{1} y+A_{1}-\beta_{1}\right)=0$, where $C_{1}>0, \alpha_{1}=\gamma_{1}=0, B_{1}>0$.

Case (a) represents a pair of vertical lines. Case (b) represents a pair of perpendicular lines with $x=0$ as one of them. This case is unbounded by the discussion in the previous section. Case (c) represents a pair consisting of the vertical line $x=0$ and a line with a negative slope in the $x y$-plane. Similarly, the reducible conic $E_{2}$ must consist of a pair of horizontal lines, a pair of perpendicular lines with $x=0$ or $y=0$ as a member or a pair consisting of the horizontal line $y=0$ and a line with a negative slope in the $x y$-plane. If none of the four lines coincide, then clearly they must intersect in at least one and at most four points in $[0, \infty)^{2}$. Some possibilities are shown in Figure 1. If one or more lines representing $E_{1}$ coincide with one or more lines representing $E_{2}$, then $E_{1}$ and $E_{2}$ must intersect in infinitely many points in $[0, \infty)^{2}$.

Next we discuss the global behavior of solutions to system (1) when it satisfies the hypotheses of Theorem 3 .

\subsection{Global behavior of solutions}

In this section, we present the proof of Theorem 3. In order to do so in a manageable way, we break up the statement of Theorem 3 into six smaller theorems based upon whether the equilibrium curves of system (1) consist of two parallel lines, two perpendicular lines, two transversal lines or some mix of the three (refer to cases (i)-(iii) at the start of Section 6).

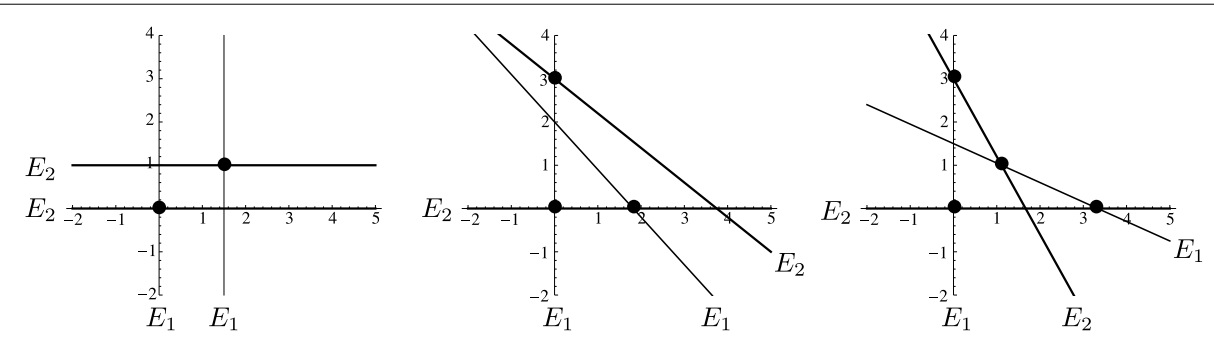

Figure 1 The dots represent equilibria when both $E_{1}$ and $E_{2}$ are reducible conics. In the third case, it is possible to have infinitely many equilibria (when the transversal lines coincide). 
In particular, we give the explicit proof for the case where both equilibrium curves are parallel lines and state the remaining five theorems, Theorems 14-18, in the Appendix at the end of this paper to avoid unnecessary repetition.

First, we present a definition and a lemma which will be required for the proof of the theorem mentioned above.

Definition 6 Recall the definition of equilibrium curves from Section 3:

$$
\begin{aligned}
& E_{1}=\left\{(x, y) \in \mathbb{R}^{2}: x=f_{1}(x, y)\right\}, \\
& E_{2}=\left\{(x, y) \in \mathbb{R}^{2}: y=f_{2}(x, y)\right\} .
\end{aligned}
$$

Consider the map $T=\left(f_{1}, f_{2}\right)$ associated to system $(1)$ restricted to the set $(0, \infty)^{2}$. Set

$$
\begin{aligned}
& R(-,-):=\left\{(x, y) \in(0, \infty)^{2}: f_{1}(x, y)<x, f_{2}(x, y)<y\right\}, \\
& R(-,+):=\left\{(x, y) \in(0, \infty)^{2}: f_{1}(x, y)<x, f_{2}(x, y)>y\right\}, \\
& R(+,-):=\left\{(x, y) \in(0, \infty)^{2}: f_{1}(x, y)>x, f_{2}(x, y)<y\right\}, \\
& R(+,+):=\left\{(x, y) \in(0, \infty)^{2}: f_{1}(x, y)>x, f_{2}(x, y)>y\right\} .
\end{aligned}
$$

Let $(\bar{x}, \bar{y})$ be an equilibrium of system (1). Denote by $Q_{\ell}, \ell=1,2,3,4$ the four regions

$$
\begin{aligned}
& Q_{1}(\bar{x}, \bar{y}):=\left\{(x, y) \in(0, \infty)^{2}: \bar{x}<x, \bar{y}<y\right\}, \\
& Q_{2}(\bar{x}, \bar{y}):=\left\{(x, y) \in(0, \infty)^{2}: \bar{x}>x, \bar{y}<y\right\}, \\
& Q_{3}(\bar{x}, \bar{y}):=\left\{(x, y) \in(0, \infty)^{2}: \bar{x}>x, \bar{y}>y\right\}, \\
& Q_{4}(\bar{x}, \bar{y}):=\left\{(x, y) \in(0, \infty)^{2}: \bar{x}<x, \bar{y}>y\right\} .
\end{aligned}
$$

Lemma 4 If the map $T:[0, \infty)^{2} \rightarrow[0, \infty)$ is competitive and possesses an interior equilibrium $(\bar{x}, \bar{y})$ which satisfies

$$
\begin{array}{ll}
Q_{1}(\bar{x}, \bar{y})=R(-,-), & Q_{2}(\bar{x}, \bar{y})=R(+,-), \\
Q_{3}(\bar{x}, \bar{y})=R(+,+), & Q_{4}(\bar{x}, \bar{y})=R(-,+),
\end{array}
$$

then $(\bar{x}, \bar{y})$ is globally asymptotically stable.

Proof By the hypotheses and the fact that any competitive map $T(x, y)$ preserves the southeast order $\preceq_{\text {se }}$, we have

$$
\begin{aligned}
(x, y) \in Q_{2}(\bar{x}, \bar{y}) \quad \Longrightarrow \quad(x, y) & \preceq_{\mathrm{se}} T(x, y) \preceq_{\mathrm{se}} T^{2}(x, y) \preceq_{\mathrm{se}} \cdots \preceq_{\mathrm{se}} T^{n}(x, y) \\
& \preceq_{\mathrm{se}} \cdots \preceq_{\mathrm{se}}(\bar{x}, \bar{y}), \\
(x, y) \in Q_{4}(\bar{x}, \bar{y}) \quad \Longrightarrow \quad(\bar{x}, \bar{y}) & \preceq_{\mathrm{se}} \cdots \preceq_{\mathrm{se}} T^{n}(x, y) \preceq_{\mathrm{se}} \cdots \preceq_{\mathrm{se}} T^{2}(x, y) \\
& \preceq_{\mathrm{se}} T(x, y) \preceq_{\mathrm{se}}(x, y) .
\end{aligned}
$$


In both cases, it follows that $T^{n}(x, y) \rightarrow(\bar{x}, \bar{y})$. Also, note that since $T$ is competitive in $(0, \infty)^{2}$, and hence in $\overline{Q_{1}(\bar{x}, \bar{y})}=\left[\bar{x}, \mathcal{U}_{1}\right] \times\left[\bar{y}, \mathcal{U}_{2}\right]$, one has

$$
\min _{(x, y) \in \overline{Q_{1}(\bar{x}, \bar{y})}} f_{1}(x, y)=f_{1}\left(\bar{x}, \mathcal{U}_{2}\right) \quad \text { and } \quad \min _{(x, y) \in \overline{Q_{1}(\bar{x}, \bar{y})}} f_{2}(x, y)=f_{2}\left(\mathcal{U}_{1}, \bar{y}\right) .
$$

Since the point $\left(\bar{x}, \mathcal{U}_{2}\right)$ lies on the line $f_{1}(x, y)=x$, one has $f_{1}\left(\bar{x}, \mathcal{U}_{2}\right)=\bar{x}$. Similarly, the point $\left(\mathcal{U}_{1}, \bar{y}\right)$ lies on the line $f_{2}(x, y)=y$ and hence $f_{2}\left(\mathcal{U}_{1}, \bar{y}\right)=\bar{y}$. It follows from this and (10) that $\overline{Q_{1}(\bar{x}, \bar{y})}$ is invariant. By a similar reasoning, one can show that $\overline{Q_{3}(\bar{x}, \bar{y})}$ is invariant. This and hypotheses (9) imply that

$$
\begin{aligned}
& (x, y) \in Q_{1}(\bar{x}, \bar{y}) \quad \Longrightarrow \quad(\bar{x}, \bar{y})<\cdots<T^{n}(x, y)<\cdots<T^{2}(x, y)<T(x, y)<(x, y), \\
& (x, y) \in Q_{3}(\bar{x}, \bar{y}) \quad \Longrightarrow \quad(x, y)<T(x, y)<T^{2}(x, y)<\cdots<T^{n}(x, y)<\cdots<(\bar{x}, \bar{y}) .
\end{aligned}
$$

Hence we have $T^{n}(x, y) \rightarrow(\bar{x}, \bar{y})$ in both these cases.

Our next theorem gives the global behavior of solutions when both equilibrium curves $E_{1}$ and $E_{2}$ of system (1) are pairs of parallel lines. It is as follows.

Theorem 5 If the graphs of $E_{1}$ and $E_{2}$ are the pairs of parallel lines

$$
\begin{aligned}
& E_{1}=\left\{(x, y) \in \mathbb{R}^{2}: B_{1} x^{2}+\left(A_{1}-\beta_{1}\right) x-\alpha_{1}=0\right\}, \\
& E_{2}=\left\{(x, y) \in \mathbb{R}^{2}: C_{2} y^{2}+\left(A_{2}-\gamma_{2}\right) y-\alpha_{2}=0\right\},
\end{aligned}
$$

then the nonnegative equilibria of system (1) and their basins of attraction must satisfy the following:

(i) If $\alpha_{1} \neq 0$ and $\alpha_{2} \neq 0$, then the unique equilibrium $\mathcal{E}_{3}$ is globally asymptotically stable.

(ii) If $\alpha_{1}=0$ and $\alpha_{2} \neq 0$, then

- If $\beta_{1}-A_{1} \leq 0$, then the unique equilibrium $\mathcal{E}_{3}$ is globally asymptotically stable.

- If $\beta_{1}-A_{1}>0$, then $\mathcal{E}_{2}$ is a saddle point with the nonnegative $y$-axis as its stable manifold. $\mathcal{E}_{3}$ is LAS and attracts all solutions with initial conditions in $(0, \infty)^{2}$ or on the positive $x$-axis.

(iii) If $\alpha_{1} \neq 0$ and $\alpha_{2}=0$, then

- If $\gamma_{2}-A_{2} \leq 0$, then the unique equilibrium $\mathcal{E}_{1}$ is globally asymptotically stable.

- If $\gamma_{2}-A_{2}>0$, then $\mathcal{E}_{1}$ is a saddle point with the nonnegative $x$-axis as its stable manifold. $\mathcal{E}_{3}$ is LAS and attracts all solutions with initial conditions in $(0, \infty)^{2}$ or on the positive $y$-axis.

(iv) If $\alpha_{1}=0$ and $\alpha_{2}=0$, then the nonnegative equilibria of system (1) and their basins of attraction must satisfy Table 3.

Proof First, suppose $\alpha_{1} \neq 0$ and $\alpha_{2} \neq 0$ in (11). Then $E_{1}$ and $E_{2}$ are given by the lines

$$
\begin{aligned}
& x=\frac{\beta_{1}-A_{1}+\sqrt{\left(\beta_{1}-A_{1}\right)^{2}+4 \alpha_{1} B_{1}}}{2 B_{1}} \text { and } \\
& y=\frac{\gamma_{2}-A_{2}+\sqrt{\left(\gamma_{2}-A_{2}\right)^{2}+4 \alpha_{2} C_{2}}}{2 C_{2}}
\end{aligned}
$$


Table 3 Global dynamics for $\alpha_{1}=0$ and $\alpha_{2}=0$ when $E_{1}$ and $E_{2}$ are pairs of parallel lines

\begin{tabular}{|c|c|c|c|c|}
\hline Parameter region & $\mathcal{E}_{0}$ & $\mathcal{E}_{1}$ & $\mathcal{E}_{2}$ & $\mathcal{E}_{3}$ \\
\hline $\begin{array}{l}\beta_{1}-A_{1}<0 \\
\gamma_{2}-A_{2}<0\end{array}$ & $\begin{array}{l}\text { G.A.S. } \\
\text { Basin of attraction: } \\
{[0, \infty)^{2}}\end{array}$ & - & - & - \\
\hline $\begin{array}{l}\beta_{1}-A_{1}>0 \\
\gamma_{2}-A_{2} \leq 0\end{array}$ & $\begin{array}{l}\text { Saddle } \\
\text { Its stable manifold: } \\
\text { Positive } y \text {-axis }\end{array}$ & $\begin{array}{l}\text { L.A.S. } \\
\text { Basin of attraction: } \\
(0, \infty)^{2} \text { and positive } \\
x \text {-axis }\end{array}$ & - & - \\
\hline $\begin{array}{l}\beta_{1}-A_{1} \leq 0 \\
\gamma_{2}-A_{2}>0\end{array}$ & $\begin{array}{l}\text { Saddle } \\
\text { Its stable manifold: } \\
\text { Positive } x \text {-axis }\end{array}$ & - & $\begin{array}{l}\text { L.A.S. } \\
\text { Basin of attraction: } \\
(0, \infty)^{2} \text { and positive } \\
y \text {-axis }\end{array}$ & - \\
\hline $\begin{array}{l}\beta_{1}-A_{1}>0 \\
\gamma_{2}-A_{2}>0\end{array}$ & Repeller & $\begin{array}{l}\text { Saddle } \\
\text { Its stable manifold: } \\
\text { Positive } x \text {-axis }\end{array}$ & $\begin{array}{l}\text { Saddle } \\
\text { Its stable manifold: } \\
\text { Positive } y \text {-axis }\end{array}$ & $\begin{array}{l}\text { L.A.S. } \\
\text { Basin of attraction: } \\
(0, \infty)^{2}\end{array}$ \\
\hline
\end{tabular}

in $[0, \infty)^{2}$. Clearly, they intersect at the unique equilibrium

$$
(\bar{x}, \bar{y})=\left(\frac{\beta_{1}-A_{1}+\sqrt{\left(\beta_{1}-A_{1}\right)^{2}+4 \alpha_{1} B_{1}}}{2 B_{1}}, \frac{\gamma_{2}-A_{2}+\sqrt{\left(\gamma_{2}-A_{2}\right)^{2}+4 \alpha_{2} C_{2}}}{2 C_{2}}\right)
$$

of system (1) which lies in $(0, \infty)^{2}$. In this case, it is easy to check that the map $T^{2}(x, y)$ is competitive and hence the unique equilibrium $(\bar{x}, \bar{y})$ is a global attractor by a result of Kulenović and Merino in [33]. Next, suppose $\alpha_{1}=0$ and $\alpha_{2} \neq 0$ in (11). Then $E_{1}$ and $E_{2}$ are given by the lines

$$
\begin{array}{ll}
E_{1}: \quad \ell_{1}: x=0, \quad \ell_{2}: x=\frac{\beta_{1}-A_{1}}{B_{1}}, \\
E_{2}: \quad y=\frac{\gamma_{2}-A_{2}+\sqrt{\left(\gamma_{2}-A_{2}\right)^{2}+4 \alpha_{2} C_{2}}}{2 C_{2}} .
\end{array}
$$

It is once again easy to check that the map $T^{2}(x, y)$ is competitive in this case. If $\beta_{1}-A_{1}>0$, then $\hat{\ell}_{1}, \hat{\ell}_{2} \in[0, \infty)^{2}$ and there exist two equilibria $\mathcal{E}_{2}=\left(0, \frac{\gamma_{2}-A_{2}+\sqrt{\left(\gamma_{2}-A_{2}\right)^{2}+4 \alpha_{2} C_{2}}}{2 C_{2}}\right)$ and $\mathcal{E}_{3}=$ $\left(\frac{\beta_{1}-A_{1}}{B_{1}}, \frac{\gamma_{2}-A_{2}+\sqrt{\left(\gamma_{2}-A_{2}\right)^{2}+4 \alpha_{2} C_{2}}}{2 C_{2}}\right)$. By Lemma $4, \mathcal{E}_{3}$ attracts every solution with initial condition in $(0, \infty)^{2}$ or on the positive $x$-axis. Moreover, since $T^{2}(x, y)$ is competitive, it is easy to check that

$$
\begin{aligned}
& (0,0) \preceq_{\text {se }} T^{2}(0,0) \preceq_{\text {se }} \cdots \preceq_{\text {se }} T^{2 n}(0,0) \preceq_{\text {se }} T^{2 n}\left(0, y_{1}\right) \preceq_{\text {se }} \mathcal{E}_{2}, \\
& \mathcal{E}_{2} \preceq_{\text {se }} T^{2 n}\left(0, y_{2}\right) \preceq_{\text {se }} T^{2 n}\left(0, \mathcal{U}_{2}\right) \preceq_{\text {se }} \cdots \preceq_{\text {se }} T^{2}\left(0, \mathcal{U}_{2}\right) \preceq_{\text {se }}\left(0, \mathcal{U}_{2}\right) .
\end{aligned}
$$

Hence we have $T^{2 n}(0,0) \rightarrow \mathcal{E}_{2}$ and $T^{2 n}\left(0, \mathcal{U}_{2}\right) \rightarrow \mathcal{E}_{2}$. As a result, $T^{2 n}(0, y) \rightarrow \mathcal{E}_{2}$ for $0<y<$ $\mathcal{U}_{2}$. Thus $\mathcal{E}_{2}$ is a saddle equilibrium with the nonnegative $y$-axis as its stable manifold.

If $\beta_{1}-A_{1} \leq 0$, then $\hat{\ell}_{2} \notin(0, \infty)^{2}$ and hence $\mathcal{E}_{2}$ is the only equilibrium in $[0, \infty)^{2}$. Note that in this case, $Q_{1}\left(\mathcal{E}_{2}\right)=\mathcal{R}(-,-)$ and $Q_{4}\left(\mathcal{E}_{2}\right)=\mathcal{R}(-,+)$. Hence, by Lemma $4, \mathcal{E}_{2}$ attracts all solutions with initial conditions in $(0, \infty)^{2}$. The proof of global attractivity of $\mathcal{E}_{2}$ for all solutions with initial conditions on the nonnegative $y$-axis is similar to the previous case. Finally, note that all solutions with initial conditions on the positive $x$-axis enter the region $(0, \infty)^{2}$ under a single application of the map $T$. 
The proof of the case $\alpha_{1} \neq 0$ and $\alpha_{2}=0$ in (11) is similar to the previous case and we skip it. Finally, suppose $\alpha_{1}=0$ and $\alpha_{2}=0$ in (11). In this case, $E_{1}$ and $E_{2}$ are given by the lines

$$
\begin{aligned}
& E_{1}: \quad \ell_{1}: x=0, \quad \ell_{2}: x=\frac{\beta_{1}-A_{1}}{B_{1}}, \\
& E_{2}: \quad \hat{\ell}_{1}: y=0, \quad \hat{\ell}_{2}: y=\frac{\gamma_{2}-A_{2}}{C_{2}} \text {. }
\end{aligned}
$$

If $\beta_{1}-A_{1} \leq 0$ and $\gamma_{2}-A_{2} \leq 0$, then $\ell_{2}, \hat{\ell}_{2} \not \subset(0, \infty)^{2}$ and the unique equilibrium $\mathcal{E}_{0}=(0,0)$ is globally asymptotically stable by Lemma 4 .

If $\beta_{1}-A_{1} \leq 0$ and $\gamma_{2}-A_{2}>0$, then $\ell_{2} \not \subset(0, \infty)^{2}$ and $\hat{\ell}_{2} \subset(0, \infty)^{2}$. Hence $\mathcal{E}_{0}$ and $\mathcal{E}_{2}=\left(0, \frac{\gamma_{2}-A_{2}}{C_{2}}\right)$ are the only equilibria present. Note that in this case, $Q_{1}\left(\mathcal{E}_{2}\right)=\mathcal{R}(-,-)$ and $Q_{4}\left(\mathcal{E}_{2}\right)=\mathcal{R}(-,+)$. Also, the dynamics of solutions with initial conditions along the positive $x$ - and $y$-axes can be determined in the same way as in the proof of the case $\alpha_{1}=0$ and $\alpha_{2} \neq 0$. The result follows from this and Lemma 4 .

If $\beta_{1}-A_{1}>0$ and $\gamma_{2}-A_{2} \leq 0$, then $\ell_{2} \subset(0, \infty)^{2}$ and $\hat{\ell}_{2} \not \subset(0, \infty)^{2}$. Hence the only equilibria present are $\mathcal{E}_{0}$ and $\mathcal{E}_{1}=\left(\frac{\beta_{1}-A_{1}}{B_{1}}, 0\right)$. This case is symmetric to the previous case and has an almost identical proof.

Finally, if $\beta_{1}-A_{1}>0$ and $\gamma_{2}-A_{2}>0$, then $\ell_{2}, \hat{\ell}_{2} \subset(0, \infty)^{2}$ and hence all four equilibria $\mathcal{E}_{0}, \mathcal{E}_{1}, \mathcal{E}_{2}$ and $\mathcal{E}_{3}=\left(\frac{\beta_{1}-A_{1}}{B_{1}}, \frac{\gamma_{2}-A_{2}}{C_{2}}\right)$ are present. In this case, global attractivity of $\mathcal{E}_{3}$ in $(0, \infty)^{2}$ is guaranteed by Lemma 4 . The proofs of the facts that $\mathcal{E}_{1}, \mathcal{E}_{2}$ are saddle equilibria with the $x$ - and $y$-axes as their stable manifolds, respectively, and that $\mathcal{E}_{0}$ is a repeller follow directly from analyzing the dynamics of solutions with initial conditions along the positive $x$ - and $y$-axes as shown in the proof of the case $\alpha_{1}=0$ and $\alpha_{2} \neq 0$. The four cases are shown in Figure 2 .

(a)

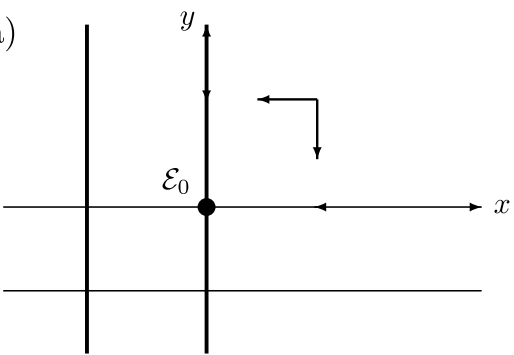

(d)

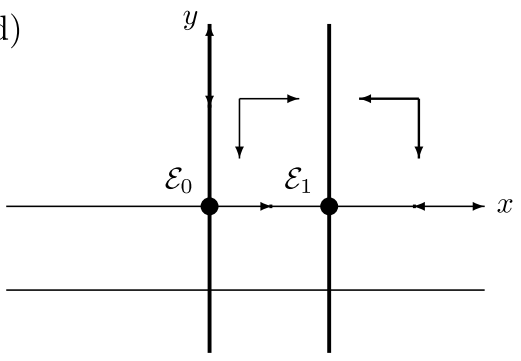

(b)

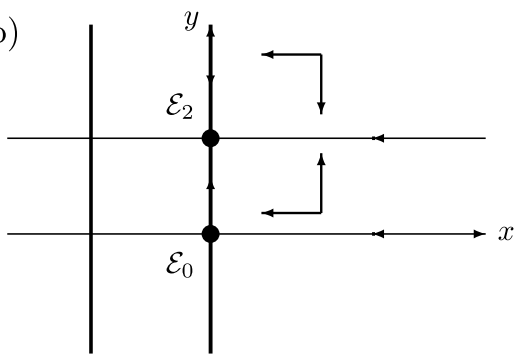

(c)

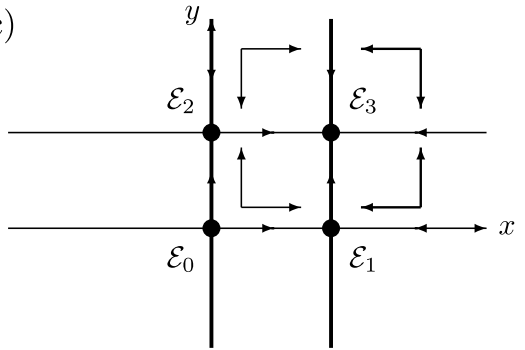

Figure 2 The figures represent attractivity of the equilibria of system (1) when (clockwise): (a) $\beta_{1}-A_{1} \leq 0$ and $\gamma_{2}-A_{2} \leq 0$, (b) $\beta_{1}-A_{1} \leq 0$ and $\gamma_{2}-A_{2}>0$, (c) $\beta_{1}-A_{1}>0$ and $\gamma_{2}-A_{2} \leq 0$, and (d) $\boldsymbol{\beta}_{1}-\boldsymbol{A}_{\mathbf{1}}>\mathbf{0}$ and $\boldsymbol{\gamma}_{\mathbf{2}}-\boldsymbol{A}_{\mathbf{2}}>\mathbf{0}$. The dark and light lines respectively represent equilibrium curves $E_{1}$ and $E_{2}$. 


\section{When exactly one of $E_{1}$ and $E_{2}$ is an irreducible conic}

In this section, we look at the case where exactly one of the equilibrium curves $E_{1}$ and $E_{2}$ of system (1) is an irreducible conic and the map $T$ associated to system (1) is bounded. Note that this case corresponds to $E_{1}$ and $E_{2}$ being combinations of pairs of parallel lines, pairs of transversal non-perpendicular lines, parabolas and hyperbolas. The cases where $E_{1}$ or $E_{2}$ is a pair of perpendicular lines are unbounded and hence not of interest to us in this paper. Thus there are $2 \times(3+2) \times(19-5)=140$ bounded members and the rest are unbounded. The next theorem is the main theorem of this section and is as follows.

Theorem 6 If system (1) is bounded and if exactly one of its equilibrium curves $E_{1}$ and $E_{2}$ is a reducible conic, that is, if either

i. $C_{1}\left(C_{1} \alpha_{1}-A_{1} \gamma_{1}\right)+\gamma_{1}\left(C_{1} \beta_{1}-B_{1} \gamma_{1}\right)=0$, or

ii. $B_{2}\left(B_{2} \alpha_{2}-A_{2} \beta_{2}\right)+\beta_{2}\left(B_{2} \gamma_{2}-C_{2} \beta_{2}\right)=0$,

then system (1) has at least one and at most two equilibria. Every solution converges to an equilibrium.

The proof of the number of equilibria is given in the next theorem. To see that every solution converges to an equilibrium, observe that in this case, exactly one member of system (1) has one of the formulas given in (i)-(iii) of the previous section. Hence exactly one of the coordinates of the map $T(x, y)$ is monotone. Thus one can use a mix of the techniques already introduced in the previous section for reducible conics along with some new techniques that will be introduced in the next section for irreducible conics to prove global convergence results for this case. We skip the proofs to avoid unnecessary repetition.

Theorem 7 If system (1) is bounded and satisfies the hypotheses of Theorem 6, then it has at least one and at most two equilibria in $[0, \infty)^{2}$. Moreover,

(a) If there exists one equilibrium, then it may be an axis equilibrium or an interior equilibrium.

(b) If there exist two equilibria, then they must include an axis equilibrium and an interior equilibrium.

(c) The set of equilibrium points must be linearly ordered by $\preceq_{\text {ne }}$.

Proof First, suppose that $E_{1}$ is an irreducible conic and $E_{2}$ is a reducible conic. Then our discussion at the start of this section implies that $E_{1}$ must have one of the following forms:

(a) $B_{1} x^{2}+\left(A_{1}-\beta_{1}\right) x-\gamma_{1} y-\alpha_{1}=0$, where $C_{1}=0, \gamma_{1}>0$;

(b) $B_{1} x^{2}+C_{1} x y+\left(A_{1}-\beta_{1}\right) x-\gamma_{1} y-\alpha_{1}=0$, where $C_{1}>0, \alpha_{1}+\gamma_{1}>0$.

In the first case, $E_{1}$ represents a parabola that opens upwards and has $x$-intercepts of opposite signs if $\alpha_{1}>0$, and a zero $x$-intercept if $\alpha_{1}=0$. In the second case, $E_{1}$ represents a hyperbola which has $x$-intercepts of opposite signs if $\alpha_{1}>0$, and a zero $x$-intercept if $\alpha_{1}=0$. This and the asymptotes of $E_{1}$ guarantee that its branch in $[0, \infty)^{2}$ is monotone. Clearly, the pair of horizontal lines representing $E_{2}$ must intersect $E_{1}$ in at least one and at most two points in $[0, \infty)^{2}$. Some possibilities are shown in Figure 3 . The monotonicity of the graph of $E_{1}$ guarantees that the set of equilibria is linearly ordered by $\preceq_{\text {ne. }}$. The proof for the case where $E_{1}$ is reducible and $E_{2}$ is nonreducible is similar and we skip it. 


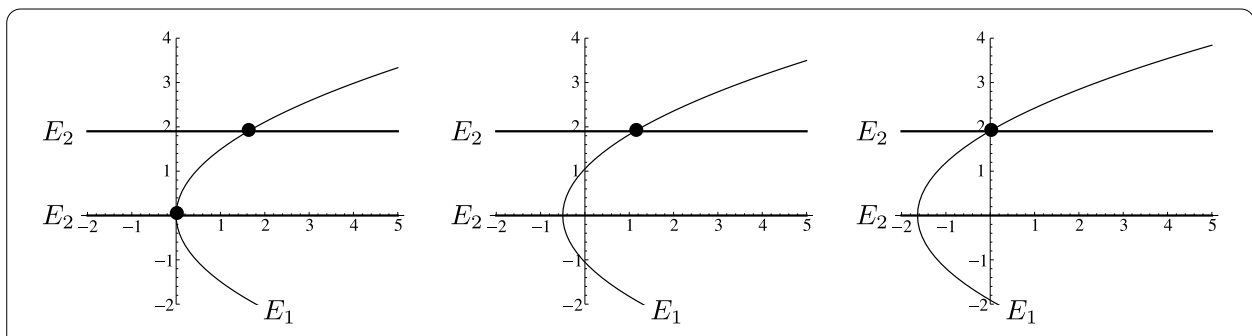

Figure 3 The dots represent equilibria when $E_{1}$ is an irreducible conic and $E_{2}$ is a reducible conic.

\section{When both $E_{1}$ and $E_{2}$ are irreducible conics}

The main theorem of this section is the following.

Theorem 8 If system (1) is bounded and if both its equilibrium curves $E_{1}$ and $E_{2}$ are irreducible conics, that is, if

i. $C_{1}\left(C_{1} \alpha_{1}-A_{1} \gamma_{1}\right)+\gamma_{1}\left(C_{1} \beta_{1}-B_{1} \gamma_{1}\right) \neq 0$, and

ii. $B_{2}\left(B_{2} \alpha_{2}-A_{2} \beta_{2}\right)+\beta_{2}\left(B_{2} \gamma_{2}-C_{2} \beta_{2}\right) \neq 0$,

then system (1) has at least one and at most three equilibria. Every solution converges to an equilibrium or to a unique minimal period-two solution which occurs as the intersection of two elliptic curves.

We present the proof of Theorem 8 at the end of Section 8.4. But first we present the number of nonnegative equilibria, local stability of equilibria, existence and uniqueness of minimal period-two solutions, and the global behavior of solutions to system (1) in Sections 8.1-8.4, respectively.

\subsection{Number of nonnegative equilibria}

We start this section by presenting a lemma which will help us establish bounds on the number of nonnegative equilibria of system (1) when both its equilibrium curves are irreducible conics.

Lemma 5 If the equilibrium curves $E_{1}$ and $E_{2}$ are irreducible conics, then all branches of the sets

$$
\begin{aligned}
& E_{1}=\left\{(x, y) \in \mathbb{R}^{2}: B_{1} x^{2}+C_{1} x y+\left(A_{1}-\beta_{1}\right) x-\gamma_{1} y-\alpha_{1}=0\right\}, \\
& E_{2}=\left\{(x, y) \in \mathbb{R}^{2}: C_{2} y^{2}+B_{2} x y+\left(A_{2}-\gamma_{2}\right) y-\beta_{2} x-\alpha_{2}=0\right\}
\end{aligned}
$$

are the graphs of monotone functions of one variable on an invariant attracting set $\mathcal{B}:=$ $\left[m_{1}, M_{1}\right] \times\left[m_{2}, M_{2}\right]$ for system (1). In particular,

(i) If $C_{1}=0$ and $B_{2}=0$, then the graphs of $E_{1}$ and $E_{2}$ are parabolas with positive slopes in $\mathcal{B}$.

(ii) If $C_{1}>0$ or $B_{2}>0$, then the graphs of $E_{1}$ and $E_{2}$ are respectively hyperbolas whose slopes in $\mathcal{B}$ have signs as given in the last two columns of Table 4 . The expression ' + or-'implies an exclusive or.

Proof First, we look at the proof of part (i). It is easy to see that when $C_{1}=0$ and $B_{2}=0$, the equilibrium curves $E_{1}$ and $E_{2}$ are parabolas opening upwards and to the right, respectively. 
Table 4 Signs of slopes of $E_{1}$ and $E_{2}$ in $\mathcal{B}$ when $C_{1}>0$ or $B_{2}>0$

\begin{tabular}{llllll}
\hline & $\boldsymbol{B}_{\boldsymbol{i}} \boldsymbol{\gamma}_{\boldsymbol{i}}-\boldsymbol{C}_{\boldsymbol{i}} \boldsymbol{\beta}_{\boldsymbol{i}}, \boldsymbol{i}=\mathbf{1 , 2}$ & $\boldsymbol{B}_{\boldsymbol{i}} \boldsymbol{\alpha}_{\boldsymbol{i}}-\boldsymbol{A}_{\boldsymbol{i}} \boldsymbol{\beta}_{\boldsymbol{i}}, \boldsymbol{i}=\mathbf{1 , 2}$ & $\boldsymbol{C}_{\boldsymbol{i}} \boldsymbol{\alpha}_{\boldsymbol{i}}-\boldsymbol{A}_{\boldsymbol{i}} \boldsymbol{\gamma}_{\boldsymbol{i}}, \boldsymbol{i}=\mathbf{1 , 2}$ & Slope of $\boldsymbol{E}_{\mathbf{1}}$ & Slope of $\boldsymbol{E}_{\mathbf{2}}$ \\
\hline (i) & $=0$ & $>0$ & $>0$ & - & + \\
(ii) & $=0$ & $<0$ & $<0$ & + & - \\
(iii) & $>0$ & $\geq 0$ & $\geq 0$ & + or - & - \\
(iv) & $>0$ & $\geq 0$ & $<0$ & + & - \\
(v) & $>0$ & $<0$ & $<0$ & + & + or - \\
(vi) & $<0$ & $\geq 0$ & $\geq 0$ & - & + or - \\
(vii) & $<0$ & $<0$ & $\geq 0$ & - & + \\
(viii) & $<0$ & $<0$ & $<0$ & + or - & + \\
\hline
\end{tabular}

Moreover, $E_{1}$ must have $x$-intercepts of opposite signs if $\alpha_{1}>0$ and a zero $x$-intercept if $\alpha_{1}=0$. Similarly, $E_{2}$ must have $y$-intercepts of opposite signs if $\alpha_{2}>0$ and a zero $y$-intercept if $\alpha_{2}=0$. Thus $E_{1}$ and $E_{2}$ must have positive slopes in $[0, \infty)^{2}$ and hence in the set $\mathcal{B}$. Next, we look at the proof of part (ii) where $C_{1}>0$ or $B_{2}>0$. We give the proof for the slopes of $E_{1}$. The proof for the slopes of $E_{2}$ is similar and we skip it. Note that $E_{1}$ can be given explicitly as a function of $x$ :

$$
E_{1}: \quad y_{1}(x)=\frac{-B_{1} x^{2}+\left(\beta_{1}-A_{1}\right) x+\alpha_{1}}{C_{1} x-\gamma_{1}}, \quad x \neq \frac{\gamma_{1}}{C_{1}} .
$$

Clearly, $E_{1}$ has a vertical asymptote $x=\frac{\gamma_{1}}{C_{1}}$ and an oblique asymptote $y=-\frac{B_{1}}{C_{1}} x-$ $\frac{A_{1} C_{1}+B_{1} \gamma_{1}-C_{1} \beta_{1}}{C_{1}^{2}}$ with a negative slope. It also has $x$-intercepts of opposite signs when $\alpha_{1}>0$, and a zero $x$-intercept when $\alpha_{1}=0$. It follows from this that the branch of $E_{1}$ which lies in $[0, \infty)^{2}$ must either lie in the region $x<\frac{\gamma_{1}}{C_{1}}$ or in the region $x>\frac{\gamma_{1}}{C_{1}}$ but not both. Moreover, it must be increasing in $x$ for $x<\frac{\gamma_{1}}{C_{1}}$ and decreasing in $x$ for $x>\frac{\gamma_{1}}{C_{1}}$. The Appendix gives that $\frac{\partial T_{1}}{\partial y}$ has constant sign which is opposite to that of $\frac{\partial T_{1}}{\partial x}$ in all cases except for cases (iii) and (viii). In all such cases, observe that

$$
\begin{aligned}
& \frac{\partial T_{1}}{\partial y}>0 \quad \Longrightarrow \quad \max _{(x, y) \in \mathcal{B}} T_{1}(x, y)=\lim _{y \rightarrow \infty} \frac{\alpha_{1}+\beta_{1} x+\gamma_{1} y}{A_{1}+B_{1} x+C_{1} y}=\frac{\gamma_{1}}{C_{1}}, \\
& \frac{\partial T_{1}}{\partial y}<0 \quad \Longrightarrow \quad \min _{(x, y) \in \mathcal{B}} T_{1}(x, y)=\lim _{y \rightarrow \infty} \frac{\alpha_{1}+\beta_{1} x+\gamma_{1} y}{A_{1}+B_{1} x+C_{1} y}=\frac{\gamma_{1}}{C_{1}} .
\end{aligned}
$$

Note that if $(\bar{x}, \bar{y})$ is an equilibrium of system (1), then it satisfies $x=T_{1}(x, y)$ and hence lies on the curve $E_{1}$. Also,

$$
\min _{(x, y) \in \mathcal{B}} T_{1}(x, y)<\bar{x}<\max _{(x, y) \in \mathcal{B}} T_{1}(x, y) .
$$

It follows from this and the previous paragraph that if $\max _{(x, y) \in \mathcal{B}} T_{1}(x, y)=\frac{\gamma_{1}}{C_{1}}$, then $\bar{x}<$ $\frac{\gamma_{1}}{C_{1}}$. Hence $E_{1}$ lies in the region $x<\frac{\gamma_{1}}{C_{1}}$ and is an increasing function of $x$. Similarly, if $\min _{(x, y) \in \mathcal{B}} T_{1}(x, y)=\frac{\gamma_{1}}{C_{1}}$, then $\bar{x}>\frac{\gamma_{1}}{C_{1}}$. Hence $E_{1}$ lies in the region $x>\frac{\gamma_{1}}{C_{1}}$ and is a decreasing function of $x$. Next consider cases (iii) and (viii) which respectively correspond to the parameter regions

(a) $B_{1} \gamma_{1}-C_{1} \beta_{1}>0, B_{1} \alpha_{1}-A_{1} \beta_{1} \geq 0, C_{1} \alpha_{1}-A_{1} \gamma_{1} \geq 0$,

(b) $B_{1} \gamma_{1}-C_{1} \beta_{1}<0, B_{1} \alpha_{1}-A_{1} \beta_{1}<0, C_{1} \alpha_{1}-A_{1} \gamma_{1}<0$.

In case (iii), the signs of $\frac{\partial T_{1}}{\partial x}$ and $\frac{\partial T_{1}}{\partial y}$ are as shown in Figure 4. 
Figure 4 The arrows indicate types of coordinatewise monotonicity of $T_{1}(x, y)$ in case (iii).

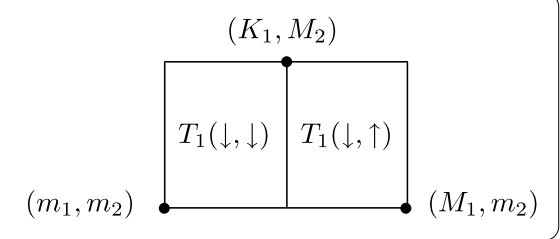

First, suppose $K_{1}<\frac{\gamma_{1}}{C_{1}}$. For all points $(x, y) \in \mathcal{B}$ with $x<K_{1}$,

$$
\min T_{1}(x, y)=\lim _{\substack{x \rightarrow K_{1} \\ y \rightarrow \infty}} \frac{\alpha_{1}+\beta_{1} x+\gamma_{1} y}{A_{1}+B_{1} x+C_{1} y}=\frac{\gamma_{1}}{C_{1}} .
$$

Moreover, for all points $(x, y) \in \mathcal{B}$ with $x>K_{1}$,

$$
\max T_{1}(x, y)=\lim _{\substack{x \rightarrow K_{1} \\ y \rightarrow \infty}} \frac{\alpha_{1}+\beta_{1} x+\gamma_{1} y}{A_{1}+B_{1} x+C_{1} y}=\frac{\gamma_{1}}{C_{1}} .
$$

Since an equilibrium $(\bar{x}, \bar{y})$ of system (1) is a fixed point that lies on the curve $E_{1}$, it follows that $\bar{x}$ must satisfy $K_{1}<\bar{x}<\frac{\gamma_{1}}{C_{1}}$. Hence $E_{1}$ must lie in the region $x<\frac{\gamma_{1}}{C_{1}}$ and must be an increasing function of $x$. One can similarly argue that if $K_{1}>\frac{\gamma_{1}}{C_{1}}$, then $E_{1}$ must be a decreasing function of $x$. Note that the case $K_{1}=\frac{\gamma_{1}}{C_{1}}$ cannot exist. Indeed, if it did, then the previous analysis would imply that the equilibrium $(\bar{x}, \bar{y})$ must lie on the line $x=K_{1}=\frac{\gamma_{1}}{C_{1}}$. But this is impossible since this line is a vertical asymptote for the curve $E_{1}$ which contains the point $(\bar{x}, \bar{y})$. In case (viii), one can use a similar proof to show that if $K_{1}<\frac{\gamma_{1}}{C_{1}}$, then $E_{1}$ is a decreasing function of $x$ and if $K_{1}>\frac{\gamma_{1}}{C_{1}}$, then $E_{1}$ is an increasing function of $x$.

Corollary 1 The following statements are true.

i. The graph of $E_{1}$ is a decreasing function of a single variable in $\mathcal{B}$ if and only if $\frac{\partial}{\partial y} T_{1}(x, y)<0$.

ii. The graph of $E_{2}$ is a decreasing function of a single variable in $\mathcal{B}$ if and only if $\frac{\partial}{\partial x} T_{2}(x, y)<0$.

The next theorem establishes bounds on the number of nonnegative equilibria of system (1).

Theorem 9 If both $E_{1}$ and $E_{2}$ are irreducible conics, then system (1) has at least one and at most three equilibria in $[0, \infty)^{2}$. In particular,

(a) If $E_{1}$ or $E_{2}$ is a parabola, then either there exists a unique interior equilibrium or there exist two equilibria, namely, $(0,0)$ and an interior equilibrium which are linearly ordered by $\preceq_{\mathrm{ne}}$.

(b) If both $E_{1}$ and $E_{2}$ are hyperbolas, then there exist between one and three equilibria all of which are interior equilibria linearly ordered by $\preceq_{\mathrm{se}}$.

Proof From the proof of part (i) of Lemma 2, it follows that when $E_{1}$ and $E_{2}$ are parabolas, their branches in $[0, \infty)^{2}$ must be increasing curves of opposite concavity, which guarantees that they must intersect at least once in $[0, \infty)^{2}$. In particular, if $\alpha_{1}=\alpha_{2}=0$, then their branches must intersect in $(0,0)$ and at an interior point of $[0, \infty)^{2}$. If $E_{1}$ is a hyperbola, 
then note that $E_{1}$ can be given explicitly as a function of $x$ :

$$
E_{1}: \quad y_{1}(x)=\frac{-B_{1} x^{2}+\left(\beta_{1}-A_{1}\right) x+\alpha_{1}}{C_{1} x-\gamma_{1}}, \quad x \neq \frac{\gamma_{1}}{C_{1}} .
$$

Clearly, $E_{1}$ has a vertical asymptote $x=\frac{\gamma_{1}}{C_{1}}$ and an oblique asymptote $y=-\frac{B_{1}}{C_{1}} x-$ $\frac{A_{1} C_{1}+B_{1} \gamma_{1}-C_{1} \beta_{1}}{C_{1}^{2}}$ with a negative slope. It also has $x$-intercepts of opposite signs when $\alpha_{1}>0$ and a zero $x$-intercept when $\alpha_{1}=0$. It follows from this that the branch of $E_{1}$ which lies in $[0, \infty)^{2}$ must lie either in the region $x<\frac{\gamma_{1}}{C_{1}}$ or in the region $x>\frac{\gamma_{1}}{C_{1}}$ but not both. Clearly, it must be increasing in the former case and decreasing in the latter case. Similarly, if $E_{2}$ is a parabola, then one can show that it must lie either in the region $y<\frac{\beta_{2}}{B_{2}}$ or in the region $y>\frac{\beta_{2}}{B_{2}}$ but not both. Also, it must be increasing in the former case and decreasing in the latter case. It follows from this that if $E_{1}$ is a parabola and $E_{2}$ is a hyperbola or vice versa, then the two must intersect in at most two points in $[0, \infty)^{2}$ including $(0,0)$ and an interior point. Moreover, if both $E_{1}$ and $E_{2}$ are hyperbolas such that one or both of them are increasing in $[0, \infty)^{2}$, then the opposite signs of their slopes/concavities guarantee that they must intersect in at most two points in $[0, \infty)^{2}$ including $(0,0)$ and an interior point. Now suppose both $E_{1}$ and $E_{2}$ are hyperbolas with decreasing branches in $[0, \infty)^{2}$. It is a consequence of Bézout's theorem (Theorem 3.1, Chapter III in [35]) that the hyperbolas $E_{1}$ and $E_{2}$ given in (4) must intersect in at most four points. Thus system (1) must have at most four equilibrium points. We claim that up to three of these four equilibrium points must lie in $B$. To see this, denote with $Q_{\ell}(a, b), \ell=1,2,3,4$ the four regions $Q_{1}(a, b):=$ $\left\{(x, y) \in R^{2}: a \leq x, b \leq y\right\}, Q_{2}(a, b):=\left\{(x, y) \in R^{2}: x \leq a, b \leq y\right\}, Q_{3}(a, b):=\left\{(x, y) \in R^{2}: x \leq\right.$ $a, y \leq b\}, Q_{4}(a, b):=\left\{(x, y) \in R^{2}: a \leq x, y \leq b\right\}$. To prove the claim, it is enough to show that $Q_{3}\left(\frac{\gamma_{1}}{C_{1}}, \frac{\beta_{2}}{B_{2}}\right)$ contains at least one equilibrium of system (1). Note that for $C_{2} \neq 0, E_{1}$ and $E_{2}$ can be given explicitly as functions of $x$ :

$$
\begin{aligned}
& E_{1}: y_{1}(x)=\frac{-B_{1} x^{2}-A_{1} x+\beta_{1} x+\alpha_{1}}{C_{1} x-\gamma_{1}}, \quad x \neq \frac{\gamma_{1}}{C_{1}}, \\
& E_{2}:\left\{\begin{array}{l}
y_{2+}(x)=\frac{-A_{2}-B_{2} x+\gamma_{2}+\sqrt{\left(-A_{2}-B_{2} x+\gamma_{2}\right)^{2}+4 C_{2}\left(\alpha_{2}+x \beta_{2}\right)}}{2 C_{2}}, \\
y_{2-}(x)=\frac{-A_{2}-B_{2} x+\gamma_{2}-\sqrt{\left(-A_{2}-B_{2} x+\gamma_{2}\right)^{2}+4 C_{2}\left(\alpha_{2}+x \beta_{2}\right)}}{2 C_{2}}
\end{array}\right.
\end{aligned}
$$

Then

$$
\lim _{x \rightarrow-\infty}\left(y_{1}(x)-y_{2-}(x)\right)=\infty \text { and } \lim _{x \rightarrow \frac{\gamma_{1}}{C_{1}}}\left(y_{1}(x)-y_{2-}(x)\right)=-\infty
$$

One can conclude from (12) and the continuity of $y_{1}(x), y_{2-}(x)$ that there exists $c<\frac{\gamma_{1}}{C_{1}}$ such that $y_{1}(c)=y_{2-}(c)$. Since $x=\frac{\gamma_{1}}{C_{1}}$ and $y=\frac{\beta_{2}}{B_{2}}$ is a horizontal asymptote of $E_{2}$, it follows from the decreasing characters of $y_{1}(x)$ and $y_{2-}(x)$ that $\left(c, y_{1}(c)\right)$ must lie in $Q_{3}\left(\frac{\gamma_{1}}{C_{1}}, \frac{\beta_{2}}{B_{2}}\right)$. When $C_{2}=0$, one can show that the equalities in (12) are still true and the conclusion follows from this. Some possible scenarios are shown in Figure 5.

\subsection{Local stability of equilibria}

In this section, we establish local stability results for the nonnegative equilibria of system (1) when both of its equilibrium curves $E_{1}$ and $E_{2}$ are irreducible conics. In particular, 

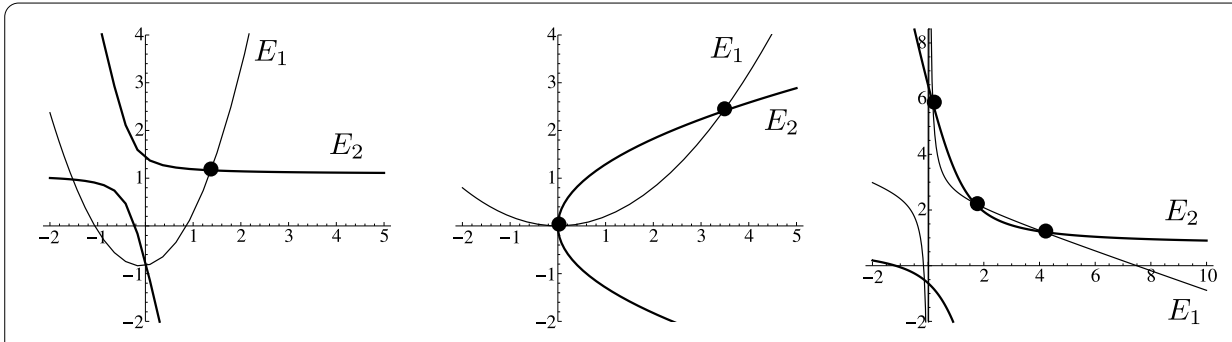

Figure 5 The dots represent equilibria when both $E_{1}$ and $E_{2}$ are irreducible conics.

we show that the local stability of the equilibria is determined by the slopes of $E_{1}$ and $E_{2}$ at these equilibria. In Theorem 9, we present local stability results when both $E_{1}$ and $E_{2}$ have negative slopes, and in Theorem 10, we do the same when at least one of them has a positive slope. We start out by giving a preliminary result on the equilibrium curves (sets) of system (1). It is a generalization of Theorem 1 in [3] and has weaker hypotheses than the latter. It also extends the latter to include the complex eigenvalues case and will be useful for proving Theorems 9 and 10.

Theorem 10 Let $R$ be a subset of $\mathbb{R}^{2}$ with a nonempty interior, and let $T=(f, g): R \rightarrow R$ be a map of class $C^{p}$ for some $p \geq 1$. Suppose that $T$ has a fixed point $(\bar{x}, \bar{y}) \in \operatorname{int} R$ such that

$$
a:=f_{x}(\bar{x}, \bar{y}), \quad b:=f_{y}(\bar{x}, \bar{y}), \quad c:=g_{x}(\bar{x}, \bar{y}), \quad d:=g_{y}(\bar{x}, \bar{y})
$$

satisfy $|a|<1$ and $|d|<1$. Let $E_{1}, E_{2}$ be the equilibrium sets

$$
E_{1}:=\{(x, y): x=f(x, y)\} \text { and } E_{2}:=\{(x, y): y=g(x, y)\}
$$

Then

i. There exists a neighborhood $I \subset \mathbb{R}$ of $\bar{x}$ and $J \subset \mathbb{R}$ of $\bar{y}$ such that the sets $E_{1} \cap(I \times J)$ and $E_{2} \cap(I \times J)$ are the graphs of class $C^{p}$ functions $y_{1}(x)$ and $y_{2}(x)$ for $x \in I$.

ii. The eigenvalues $\lambda_{1}$ and $\lambda_{2}$ of the Jacobian matrix of $T$ at $(\bar{x}, \bar{y})$ satisfy:

(a) If $\lambda_{1}, \lambda_{2}$ are real and equal, then $-1<\lambda_{1}, \lambda_{2}<1$.

(b) If $\lambda_{1}, \lambda_{2}$ are real and distinct with $\lambda_{2}<\lambda_{1}$, then $-1<\lambda_{1}$ and $\lambda_{2}<1$. Furthermore, $b \neq 0$ and

$$
\operatorname{sign}\left(1+\lambda_{2}\right)=\operatorname{sign}(1+a+d+a d-b c)
$$

and

$$
\operatorname{sign}\left(1-\lambda_{1}\right)= \begin{cases}-\operatorname{sign}\left(y_{1}^{\prime}(\bar{x})-y_{2}^{\prime}(\bar{x})\right) & \text { if } b<0, \\ \operatorname{sign}\left(y_{1}^{\prime}(\bar{x})-y_{2}^{\prime}(\bar{x})\right) & \text { if } b>0 .\end{cases}
$$

(c) If $\lambda_{1}$ and $\lambda_{2}$ are complex numbers, then

$$
\bar{\lambda}_{1}=\lambda_{2} \quad \text { and } \quad-1<\left|\lambda_{1}\right|=\left|\lambda_{2}\right|<1 \text {. }
$$


i. The existence of $I$ and $J$ and of smooth functions $y_{1}(x)$ and $y_{2}(x)$ defined in $I$ as in the statement of the theorem is guaranteed by the hypotheses and the implicit function theorem. Moreover, when $f_{y}(x, y) \neq 0$, one has

$$
y_{1}^{\prime}(x)=\frac{1-f_{x}(x, y)}{f_{y}(x, y)} \quad \text { and } \quad y_{2}^{\prime}(x)=\frac{g_{x}(x, y)}{1-g_{y}(x, y)}, \quad x \in I
$$

Note that $f_{y}(x, y) \neq 0$ since otherwise one would have $f_{x}(x, y)=1$ in (17) upon rewriting the first expression as $f_{y}(x, y) y_{1}^{\prime}(x)=1-f_{x}(x, y)$ and thus $a:=f_{x}(\bar{x}, \bar{y})=1$, contradicting one of the hypotheses of the theorem.

ii. The characteristic polynomial of the Jacobian of $T$,

$$
p(\lambda)=\lambda^{2}-(a+d) \lambda+(a d-b c)
$$

has $\lambda_{1}$ and $\lambda_{2}$ as its roots. If $\lambda_{1}=\lambda_{2}=\lambda$, then the hypotheses $-1<a<1$ and $-1<d<1$ and the sum-of-roots relation for quadratic functions applied to (18) imply

$$
-2<2 \lambda=a+d<2 \quad \Longrightarrow \quad-1<\lambda<1 \text {, }
$$

which proves (a). Now, suppose $\lambda_{1}, \lambda_{2}$ are real and distinct with $\lambda_{2}<\lambda_{1}$. Since $-2<a+d=\lambda_{1}+\lambda_{2}<2$, the larger root $\lambda_{1}$ must satisfy $-1<\lambda_{1}$ and the smaller root $\lambda_{2}$ must satisfy $\lambda_{2}<1$. Moreover, the remark following (17) in part i gives that $b:=f_{y}(\bar{x}, \bar{y}) \neq 0$. To see the proof of (14), note that in (18), we have $p(-1)=1+(a+d)+a d-b c=\left(-1-\lambda_{1}\right)\left(-1-\lambda_{2}\right)$. Since $-1<\lambda_{1}$ from above, it follows that $p(-1)>0$ if and only if $-1-\lambda_{2}<0$, that is, if and only if $1+\lambda_{2}>0$. Next note that from (17), we have

$$
\begin{aligned}
y_{1}^{\prime}(\bar{x})-y_{2}^{\prime}(\bar{x}) & =\frac{1-a}{b}-\frac{c}{1-d}=\frac{1-(a+d)+a d-b c}{b(1-d)} \\
& =\frac{p(1)}{b(1-d)}=\frac{\left(1-\lambda_{1}\right)\left(1-\lambda_{2}\right)}{b(1-d)} .
\end{aligned}
$$

The proof of (15) is a direct consequence of (19), the inequality $\lambda_{2}<1$ and the hypothesis $|d|<1$. Next suppose that $\lambda_{1}, \lambda_{2}$ are complex numbers. Clearly, $\bar{\lambda}_{1}=\lambda_{2}$ in this case. From (18), we have

$$
\lambda_{1}, \lambda_{2}=\frac{a+d \pm i \sqrt{(a+d)^{2}-4(a d-b c)}}{2} .
$$

Note that a necessary condition for the discriminant to be negative is $b c<0$ since it can be rewritten as $(a-d)^{2}+4 b c$. It follows from this and the hypotheses $|a|<1$ and $|d|<1$ that

$$
\left|\lambda_{1}\right|^{2}=\left|\lambda_{2}\right|^{2}=\frac{a^{2}+d^{2}+2 b c}{2}<\frac{a^{2}+d^{2}}{2}<1
$$

Corollary 2 If $\left(y_{1}^{\prime}(\bar{x})-y_{2}^{\prime}(\bar{x})\right) b>0$, then system (1) cannot possess any repelling fixed points. 
This is a direct consequence of Theorem 10 part ii.(b) since it is clear from (15) that under the given hypothesis, $1>\lambda_{1}$. Next, we give a complete description of the local behavior of the equilibria of system (1). Recall that the map $T(x, y)=\left(T_{1}(x, y), T_{2}(x, y)\right)$ associated with system (1) is

$$
T(x, y)=\left(\frac{\alpha_{1}+\beta_{1} x+\gamma_{1} y}{A_{1}+B_{1} x+C_{1} y}, \frac{\alpha_{2}+\beta_{2} x+\gamma_{2} y}{A_{2}+B_{2} x+C_{2} y}\right), \quad(x, y) \in[0, \infty) \times[0, \infty) .
$$

For future reference, we give the Jacobian matrix of $T$ at $(x, y)$ :

$$
\begin{aligned}
J_{T}(x, y) & =\left(\begin{array}{ll}
f_{1}(x, y) & f_{1}(x, y) \\
f_{2 x}(x, y) & f_{2 y}(x, y)
\end{array}\right) \\
& =\left(\begin{array}{ll}
\frac{-B_{1} \alpha_{1}+A_{1} \beta_{1}+C_{1} \beta_{1} y-B_{1} \gamma_{1} y}{\left(A_{1}+B_{1} x+C_{1} y\right)^{2}} & \frac{-C_{1} \alpha_{1}+A_{1} \gamma_{1}-C_{1} \beta_{1} x+B_{1} \gamma_{1} x}{\left(A_{1}+B_{1} x+C_{1} y\right)^{2}} \\
\frac{-B_{2} \alpha_{2}+A_{2} \beta_{2}+C_{2} \beta_{2} y-B_{2} \gamma_{2} y}{\left(A_{2}+B_{2} x+C_{2} y\right)^{2}} & \frac{-C_{2} \alpha_{2}+A_{2} \gamma_{2}-C_{2} \beta_{2} x+B_{2} \gamma_{2} x}{\left(A_{2}+B_{2} x+C_{2} y\right)^{2}}
\end{array}\right) .
\end{aligned}
$$

The next lemma gives a connection between the slopes of equilibrium curves $E_{1}, E_{2}$ in the invariant attracting box $\mathcal{B}$ and the signs of entries of the Jacobian in (21) evaluated at an equilibrium point of (1).

Lemma 6 The map T satisfies the hypotheses of Theorem 10.

Proof Set $a:=f_{1_{x}}(\bar{x}, \bar{y}), b:=f_{1_{y}}(\bar{x}, \bar{y}), c:=f_{2_{x}}(\bar{x}, \bar{y}), d:=f_{2_{y}}(\bar{x}, \bar{y})$. Implicit differentiation of the equations defining $E_{1}$ and $E_{2}$ in $(13)$ at $(\bar{x}, \bar{y})$ gives

$$
y_{1}^{\prime}(\bar{x})=\frac{1-a}{b} \quad \text { and } \quad y_{2}^{\prime}(\bar{x})=\frac{c}{1-d}
$$

It is a direct consequence of Lemma 5 and Corollary 1 that $a<1$ and $d<1$ in (22). Next note that the fixed point $(\bar{x}, \bar{y})$ must satisfy $T(\bar{x}, \bar{y})=(\bar{x}, \bar{y})$. Taking the difference in this equality and solving for $\alpha_{1}$ and $\alpha_{2}$ in the numerators, we get

$$
\alpha_{1}=B_{1} \bar{x}^{2}+A_{1} \bar{x}+C_{1} \overline{x y}-\beta_{1} \bar{x}-\gamma_{1} \bar{y} \quad \text { and } \quad \alpha_{2}=C_{2} \bar{y}^{2}+A_{2} \bar{y}+B_{2} \overline{x y}-\beta_{2} \bar{x}-\gamma_{2} \bar{y} .
$$

Replacing $\alpha_{1}$ and $\alpha_{2}$ in the expressions for $1+a$ and $1+d$ by their equivalent expressions from (23), we get

$$
1+a=\frac{A_{1}+C_{1} \bar{y}+\beta_{1}}{A_{1}+B_{1} \bar{x}+C_{1} \bar{y}} \quad \text { and } \quad 1+d=\frac{A_{2}+B_{2} \bar{x}+\gamma_{2}}{A_{2}+B_{2} \bar{x}+C_{2} \bar{y}}
$$

which are clearly positive. It follows that $-1<a<1$ and $-1<d<1$.

Theorem 11 If the graphs of both $E_{1}$ and $E_{2}$ are decreasing functions of a single variable in the invariant attracting set $B$, then the following statements are true.

(i) System (1) has at least one and at most three equilibria in $(0, \infty)^{2}$. The set of equilibrium points is linearly ordered by $\preceq_{\text {se }}$.

(ii) If system (1) has exactly one equilibrium in $(0, \infty)^{2}$, then it is locally asymptotically stable. If $(0,0)$ is an equilibrium, then it is a repeller. 
(iii) If system (1) has three distinct equilibria in $(0, \infty)^{2}$, say $\left(\bar{x}_{\ell}, \bar{y}_{\ell}\right), l=1, \ldots, 3$, with $\left(\bar{x}_{1}, \bar{y}_{1}\right) \preceq_{\text {se }}\left(\bar{x}_{2}, \bar{y}_{2}\right) \preceq_{\text {se }}\left(\bar{x}_{3}, \bar{y}_{3}\right)$, then $\left(\bar{x}_{1}, \bar{y}_{1}\right)$ and $\left(\bar{x}_{3}, \bar{y}_{3}\right)$ are locally asymptotically stable, while $\left(\bar{x}_{2}, \bar{y}_{2}\right)$ is a saddle point.

(iv) If there exist exactly two equilibria in $(0, \infty)^{2}$, then one is locally asymptotically stable and the other is a nonhyperbolic equilibrium.

Proof First, observe that the eigenvalues $\lambda_{1}, \lambda_{2}$ of $T$ are roots of characteristic equation (18) of the Jacobian matrix of $T$. A sufficient condition for the discriminant of (18) to be positive is $b c>0$, which is guaranteed by Corollary 1 and the hypothesis of the theorem. It follows that $\lambda_{1}, \lambda_{2}$ are real and distinct. Next, note that by (23) we have

$$
\begin{aligned}
1+ & (a+d)+a d-b c \\
= & \left(A_{1} A_{2}+C_{1} A_{2} \bar{y}+\beta_{1} A_{2}+A_{1} B_{2} \bar{x}+B_{2} \beta_{1} \bar{x}+C_{1} \beta_{2} \bar{x}\right. \\
& \left.+\gamma_{1}\left(B_{2} \bar{y}-\beta_{2}\right)+A_{1} \gamma_{2}+C_{1} \bar{y} \gamma_{2}+\beta_{1} \gamma_{2}\right) \\
& /\left(\left(A_{1}+B_{1} \bar{x}+C_{1} \bar{y}\right)\left(A_{2}+B_{2} \bar{x}+C_{2} \bar{y}\right)\right),
\end{aligned}
$$

which is positive by the inequality $\bar{y}>\frac{\beta_{2}}{B_{2}}$ since $(\bar{x}, \bar{y})$ lies on the decreasing curve $E_{2}$ with a horizontal asymptote at $y=\frac{\beta_{2}}{B_{2}}$. Hence, in (14), $\lambda_{2}>-1$. It follows from Theorem 10 part ii.(b) that $-1<\lambda_{2}<1$.

The proofs of parts (i)-(iv) are given below.

(i) This is direct consequence of Theorem 9.

(ii) Solving for $y$ and $x$ respectively in the equations defining $E_{1}$ and $E_{2}$ in (4) gives that the vertical asymptote of $E_{1}$ is $x=\frac{\gamma_{1}}{C_{1}}$ and the horizontal asymptote of $E_{2}$ is $y=\frac{\beta_{2}}{B_{2}}$. The asymptotes guarantee that in order to have exactly one intersection point $(\bar{x}, \bar{y})$ in $[0, \infty)^{2}$, the slopes of the functions $y_{1}(x)$ and $y_{2}(x)$ of $E_{1}$ and $E_{2}$, respectively, must satisfy the relation $y_{1}^{\prime}(\bar{x})<y_{2}^{\prime}(\bar{x})$. Theorem 10 part ii.(b) then gives that $(\bar{x}, \bar{y})$ must be locally asymptotically stable.

(iii) The asymptotes guarantee that in order to have three intersection points in $[0, \infty)^{2}$, the slopes of the functions $y_{1}(x)$ and $y_{2}(x)$ of $E_{1}$ and $E_{2}$, respectively, must satisfy the relations $y_{1}^{\prime}\left(\bar{x}_{1}\right)<y_{2}^{\prime}\left(\bar{x}_{1}\right), y_{1}^{\prime}\left(\bar{x}_{2}\right)>y_{2}^{\prime}\left(\bar{x}_{2}\right)$ and $y_{1}^{\prime}\left(\bar{x}_{3}\right)<y_{2}^{\prime}\left(\bar{x}_{3}\right)$. It then follows from Theorem 10 part ii.(b) that $\left(\bar{x}_{1}, \bar{y}_{1}\right)$ and $\left(\bar{x}_{3}, \bar{y}_{3}\right)$ must be locally asymptotically stable, while $\left(\bar{x}_{2}, \bar{y}_{2}\right)$ must be a saddle point.

(iv) The asymptotes guarantee that in order to have two intersection points in $[0, \infty)^{2}$, the graphs of $E_{1}$ and $E_{2}$ must have exactly one transversal intersection point $\left(\bar{x}_{1}, \bar{y}_{1}\right)$ with $y_{1}^{\prime}\left(\bar{x}_{1}\right)<y_{2}^{\prime}\left(\bar{x}_{1}\right)$. It follows that the remaining intersection point $\left(\bar{x}_{2}, \bar{y}_{2}\right)$ must be tangential in nature, with $y_{1}^{\prime}\left(\bar{x}_{2}\right)=y_{2}^{\prime}\left(\bar{x}_{2}\right)$. Theorem 10 part ii.(b) then gives that $\left(\bar{x}_{1}, \bar{y}_{1}\right)$ must be locally asymptotically stable and $\left(\bar{x}_{2}, \bar{y}_{2}\right)$ must be a nonhyperbolic equilibrium.

Theorem 12 If the graph of at least one of $E_{1}$ and $E_{2}$ is an increasing function of a single variable in the invariant attracting set $B$, then the following statements are true.

(i) System (1) has a unique interior equilibrium $(\bar{x}, \bar{y})$ in $[0, \infty)^{2}$.

(ii) If the graph of exactly one of $E_{1}$ and $E_{2}$ is an increasing function of a single variable in $B$, then $(\bar{x}, \bar{y})$ is locally asymptotically stable. 
(iii) If the graphs of both $E_{1}$ and $E_{2}$ are increasing functions of a single variable in $B$, then $(\bar{x}, \bar{y})$ is either locally asymptotically stable or nonhyperbolic or a saddle point equilibrium. In particular,

1. If $1+(a+d)+a d-b c>0$, then $(\bar{x}, \bar{y})$ is locally asymptotically stable.

2. If $1+(a+d)+a d-b c=0$, then $(\bar{x}, \bar{y})$ is a nonhyperbolic equilibrium.

3. If $1+(a+d)+a d-b c<0$, then $(\bar{x}, \bar{y})$ is a saddle point equilibrium.

Proof

(i) This follows directly from Theorem 9.

(ii) From the hypothesis and Corollary 1, it follows that $b c<0$ in (18) and hence the latter possesses real distinct roots. Theorem 10 part ii.(b) then gives that $(\bar{x}, \bar{y})$ must be locally asymptotically stable.

(iii) From the hypothesis and Corollary 1, one must have $b>0, c>0$ and hence $b c>0$ in (18). If the discriminant of the latter is negative or zero, then part ii.(a) and part ii.(c) of Theorem 10 give that $(\bar{x}, \bar{y})$ is locally asymptotically stable. If the discriminant of (18) is positive, then the hypothesis and the asymptotes of $E_{1}$ and $E_{2}$ guarantee that $y_{1}^{\prime}(\bar{x})>y_{2}^{\prime}(\bar{x})$ and hence $1>\lambda_{1}$ in the second part of (15). The rest of the proof follows from Theorem 10 part ii.(b).

\subsection{Existence and uniqueness of minimal period-two solutions}

Here we look at minimal period-two solutions of system (1). In particular, we show that if system (1) possesses a minimal period-two solution in the nonnegative quadrant, then this minimal period-two solution must be unique. The main theorem of this section is as follows.

Theorem 13 System (1) possesses a unique minimal period-two solution in $\mathbb{R}_{+}^{2}$ which exists if and only if there are no multiple equilibria. When the minimal period-two solution exists, the unique equilibrium is a saddle point.

The proof of the theorem follows from the statements of Propositions 1 and 2 given below. But first we present a lemma that gives important geometrical properties of the minimal period-two solutions of system (1).

Lemma 7 The minimal period-two points of system (1) are intersection points of decreasing branches of elliptic curves in $\mathbb{R}_{+}^{2}$. Moreover, each branch has at most one inflection point in $\mathbb{R}_{+}^{2}$.

Proof Note that period-two solutions of (1) must satisfy the equation $T^{2}(x, y)=(x, y)$, where $(x, y) \in[0, \infty)^{2}$. The latter on simplification gives rise to a system of equations having the general form shown below where the coefficients in the first equation are functions of the parameters $\alpha_{1}, \beta_{1}, \gamma_{1}, A_{1}, B_{1}$ and $C_{1}$, and the coefficients in the second equation are functions of the parameters $\alpha_{2}, \beta_{2}, \gamma_{2}, A_{2}, B_{2}$ and $C_{2}$.

$$
\begin{array}{ll}
\mathcal{E}_{1}: & A x^{3}+B x^{2} y+C x y^{2}+E x^{2}+F x y+G y^{2}+H x+I y+J=0, \\
\mathcal{E}_{2}: & \widetilde{B} x^{2} y+\widetilde{C} x y^{2}+\widetilde{D} y^{3}+\widetilde{E} x^{2}+\widetilde{F} x y+\widetilde{G} y^{2}+\widetilde{H} x+\widetilde{I} y+\widetilde{J}=0 .
\end{array}
$$

The solution set of each equation in (24) belongs to an elliptic curve defined over the field of reals $\mathbb{R}$. To see that the branches of $\mathcal{E}_{1}$ and $\mathcal{E}_{2}$ are decreasing in $\mathbb{R}_{+}^{2}$, observe that the 
Figure 6 Shapes of the elliptic curves $\mathcal{E}_{1}$ and $\mathcal{E}_{2}$.

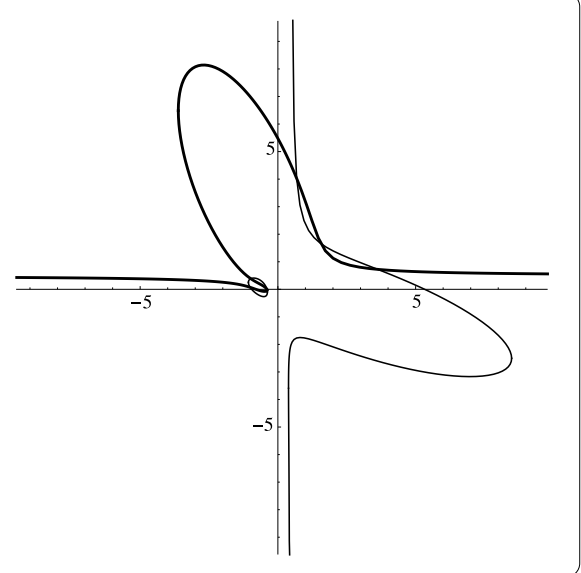

two equations in (24) are respectively quadratic equations in $y$ and $x$. Solving for $y$ and $x$ respectively in these two equations, we get that the positive branches of the curves $\mathcal{E}_{1}$ and $\mathcal{E}_{2}$ are graphs of injective functions of $x$ and $y$, respectively. Hence they are either increasing or decreasing in $\mathbb{R}_{+}^{2}$. The proof of the first part of the lemma follows from this, the observation that minimal period-two solutions are precisely the intersection points of $\mathcal{E}_{1}$ and $\mathcal{E}_{2}$ and the fact from the last subsection that minimal period-two points of system (1) are always ordered by the south-east ordering $\preceq_{\text {se }}$. To see the proof of the second part, note that a well-known property of elliptic curves says that any straight line joining two inflection points on an elliptic curve must contain a third one (see [43-45]). This property, the decreasing natures of the two elliptic curves $\mathcal{E}_{1}$ and $\mathcal{E}_{2}$ and their respective vertical and horizontal asymptotes guarantee the existence of at most one inflection point for each in $\mathbb{R}_{+}^{2}$. The shapes of $\mathcal{E}_{1}$ and $\mathcal{E}_{2}$ are shown in Figure 6.

Proposition 1 System (1) has a unique minimal period-two solution in $\mathbb{R}_{+}^{2}$. The minimal period-two solution cannot coexist with multiple equilibria.

Proof From the statement of the previous lemma, it follows that one of two cases is possible: (i) at least one of the two elliptic curves $\mathcal{E}_{1}$ and $\mathcal{E}_{2}$ has an inflection point in $\mathbb{R}_{+}^{2}$, or (ii) none of the two elliptic curves $\mathcal{E}_{1}$ and $\mathcal{E}_{2}$ has inflection points in $\mathbb{R}_{+}^{2}$. One can easily see that in the first case, the two curves must intersect in at most three points in $\mathbb{R}_{+}^{2}$. In the second case, it is not hard to see that the existence of the fourth intersection point either requires $\mathcal{E}_{1}$ to increase at some point or requires $\mathcal{E}_{2}$ to have an inflection point in $\mathbb{R}_{+}^{2}$. The first requirement contradicts the statement of the previous lemma and the second requirement goes against the hypothesis of case (ii). The three possibilities are shown in Figure 7. To see that minimal period-two solutions and multiple equilibria cannot coexist in $\mathbb{R}_{+}^{2}$, recall that a necessary condition for multiple equilibria to exist is that both equilibrium curves of system (1) must be decreasing in nature. One can easily check that in all parameter regions from the last subsection where minimal period-two solutions may exist, one or both equilibrium curves are increasing in nature.

Proposition 2 If system (1) possesses a minimal period-two solution in $\mathbb{R}_{+}^{2}$, then its unique equilibrium must be a saddle point. 

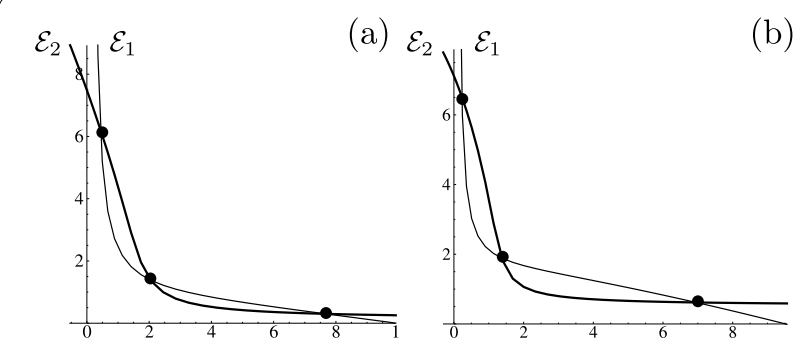

(b)

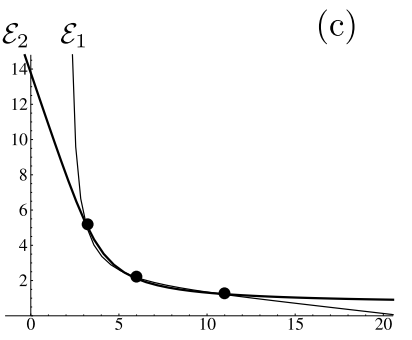

Figure 7 The dots represent minimal period-two solutions when (a) $\mathcal{E}_{2}$ has an inflection point, (b) both $\mathcal{E}_{1}$ and $\mathcal{E}_{2}$ have inflection points, and (c) neither $\mathcal{E}_{1}$ nor $\mathcal{E}_{2}$ has an inflection point.

Proof We saw in Lemma 7 that the minimal period-two points of system (1) are intersection points of decreasing branches of the elliptic curves $\mathcal{E}_{1}$ and $\mathcal{E}_{2}$ in $\mathbb{R}_{+}^{2}$. We also saw in the last subsection that the two minimal period-two points, say $P_{0}$ and $P_{1}$, and the unique equilibrium $(\bar{x}, \bar{y})$ are ordered by the south-east ordering as follows: $P_{0} \preceq_{\text {se }}(\bar{x}, \bar{y}) \preceq_{\text {se }} P_{1}$. Consider the open region enclosed by $P_{0},(\bar{x}, \bar{y})$ and the decreasing elliptic curves $\mathcal{E}_{1}$ and $\mathcal{E}_{2}$. Let $T^{2}(x, y):=\left(\tau_{1}(x, y), \tau_{2}(x, y)\right)$. Since $\mathcal{E}_{1}$ and $\mathcal{E}_{2}$ respectively have formulas $\tau_{1}(x, y)=x$ and $\tau_{2}(x, y)=y$, one must have $\tau_{1}(x, y)<x$ and $\tau_{2}(x, y)>y$ for $(x, y)$ in this region. It follows that $T^{2}(x, y) \preceq_{\text {se }}(x, y) \preceq_{\text {se }}(\bar{x}, \bar{y})$ here. If $T^{2}(x, y)$ escapes this region, then the proof is complete. Otherwise, one can always keep iterating until either $T^{2 n}(x, y) \rightarrow P_{0}$ or $T^{2 n}(x, y)$ lies outside the region for some $n$.

\subsection{Global behavior of solutions}

In this section, we discuss global behavior of solutions to system (1) when both its equilibrium curves $E_{1}$ and $E_{2}$ are irreducible conics. Before presenting the main theorem of this section, we define a nested invariant attracting set for system (1) which will be key to the proof of this theorem.

Definition 7 Suppose that the bounded map $T(x, y)=\left(T_{1}(x, y), T_{2}(x, y)\right)$ satisfies $m_{1} \leq$ $T_{1}(x, y) \leq M_{1}$ and $m_{2} \leq T_{2}(x, y) \leq M_{2}$. Define

$$
\left.\begin{array}{l}
\mathcal{L}_{i}:=\min \left\{T_{i}(x, y):(x, y) \in\left[m_{1}, M_{1}\right] \times\left[m_{2}, M_{2}\right]\right\} \\
\mathcal{U}_{i}:=\max \left\{T_{i}(x, y):(x, y) \in\left[m_{1}, M_{1}\right] \times\left[m_{2}, M_{2}\right]\right\}
\end{array}\right\}, \quad i=1,2 .
$$

Remark Note that $T\left(\left[m_{1}, M_{1}\right] \times\left[m_{2}, M_{2}\right]\right) \subseteq\left[\mathcal{L}_{1}, \mathcal{U}_{1}\right] \times\left[\mathcal{L}_{2}, \mathcal{U}_{2}\right] \subseteq\left[m_{1}, M_{1}\right] \times\left[m_{2}, M_{2}\right]$

The next lemma gives explicit formulas for $\mathcal{L}_{1}, \mathcal{U}_{1}, \mathcal{L}_{2}$ and $\mathcal{U}_{2}$ for different parameter regions of system (1).

Lemma 8 The formulas for $\mathcal{L}_{1}, \mathcal{U}_{1}, \mathcal{L}_{2}$ and $\mathcal{U}_{2}$ for different parameter regions are as shown in Table 5.

Proof In case 1, it is easy to check that for $i=1, \frac{\partial T_{1}}{\partial x}<0$ and $\frac{\partial T_{1}}{\partial y}<0$ for $(x, y) \in \mathcal{B}$. Since $T_{1}(x, y)$ is nonincreasing in $x$ and $y$ on $\mathcal{B}$, hence $T_{1}\left(M_{1}, M_{2}\right) \leq T_{1}(x, y) \leq T_{1}\left(m_{1}, m_{2}\right)$. Choose $\mathcal{L}_{1}=T_{1}\left(M_{1}, M_{2}\right)$ and $\mathcal{U}_{1}=T_{1}\left(m_{1}, m_{2}\right)$. One can similarly show that for $i=2$, $\mathcal{L}_{2}=T_{2}\left(M_{1}, M_{2}\right)$ and $\mathcal{U}_{2}=T_{2}\left(m_{1}, m_{2}\right)$. The proof of case 2 is similar and we skip it. In case 3, Definition 5 and Lemma 2 give that the signs of the partial derivatives of $T_{1}(x, y)$ 
Table 5 Table of formulas for $\mathcal{L}_{1}, \mathcal{U}_{1}, \mathcal{L}_{2}$ and $\mathcal{U}_{2}$

\begin{tabular}{llllll}
\hline & $\boldsymbol{B}_{\boldsymbol{i}} \boldsymbol{\gamma}_{\boldsymbol{i}}-\boldsymbol{C}_{\boldsymbol{i}} \boldsymbol{\beta}_{\boldsymbol{i}}, \boldsymbol{i}=\mathbf{1 , 2}$ & $\boldsymbol{B}_{\boldsymbol{i}} \boldsymbol{\alpha}_{\boldsymbol{i}}-\boldsymbol{A}_{\boldsymbol{i}} \boldsymbol{\beta}_{\boldsymbol{i}}, \boldsymbol{i}=\mathbf{1 , 2}$ & $\boldsymbol{C}_{\boldsymbol{i}} \boldsymbol{\alpha}_{\boldsymbol{i}}-\boldsymbol{A}_{\boldsymbol{i}} \boldsymbol{\gamma}_{\boldsymbol{i}}, \boldsymbol{i}=\mathbf{1 , 2}$ & $\mathcal{L}_{\boldsymbol{i}}, \boldsymbol{i}=\mathbf{1}, \mathbf{2}$ & $\mathcal{U}_{\boldsymbol{i}}, \boldsymbol{i}=\mathbf{1 , \boldsymbol { 2 }}$ \\
\hline 1. & $=0$ & $>0$ & $T_{i}\left(M_{1}, M_{2}\right)$ & $T_{i}\left(m_{1}, m_{2}\right)$ \\
2. & $>0$ & $<0$ & $T_{i}\left(m_{1}, m_{2}\right)$ & $T_{i}\left(M_{1}, M_{2}\right)$ \\
3. & $>0$ & $\geq 0$ & $\geq 0$ & $T_{i}\left(M_{1}, m_{2}\right)$ & $T_{i}\left(m_{1}, m_{2}\right)$ \\
4. & $>0$ & $>0$ & $<0$ & $T_{i}\left(M_{1}, m_{2}\right)$ & $T_{i}\left(m_{1}, M_{2}\right)$ \\
5. & $>0$ & $<0$ & $<0$ & $T_{i}\left(m_{1}, m_{2}\right)$ & $T_{i}\left(m_{1}, M_{2}\right)$ \\
6. & $<0$ & $>0$ & $\geq 0$ & $T_{i}\left(m_{1}, M_{2}\right)$ & $T_{i}\left(m_{1}, m_{2}\right)$ \\
7. & $<0$ & $\geq 0$ & $\geq 0$ & $T_{i}\left(m_{1}, M_{2}\right)$ & $T_{i}\left(M_{1}, m_{2}\right)$ \\
8. & $<0$ & $<0$ & $<0$ & $T_{i}\left(m_{1}, m_{2}\right)$ & $T_{i}\left(M_{1}, m_{2}\right)$ \\
\hline
\end{tabular}

Figure 8 The arrows indicate types of coordinatewise monotonicity of $T_{1}(x, y)$ in case 3 .

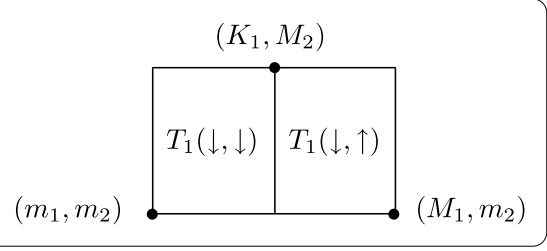

are constant on the interior of each of the sets $\left[m_{1}, K_{1}\right] \times\left[m_{2}, M_{2}\right]$ and $\left[K_{1}, M_{1}\right] \times\left[m_{2}, M_{2}\right]$. This is illustrated in Figure 8.

Then $T_{1}\left(M_{1}, m_{2}\right) \leq T_{1}(x, y) \leq T_{1}\left(K_{1}, M_{2}\right)$. Similarly, $T_{1}(x, y)$ is nonincreasing in both $x$ and $y$ on $\left[m_{1}, K_{1}\right] \times\left[m_{2}, M_{2}\right]$ and hence $T_{1}\left(K_{1}, M_{2}\right) \leq T_{1}(x, y) \leq T_{1}\left(m_{1}, m_{2}\right)$. It follows from these two observations that for $(x, y) \in\left[m_{1}, M_{1}\right] \times\left[m_{2}, M_{2}\right]$, one must have $T_{1}\left(M_{1}, m_{2}\right) \leq$ $T_{1}(x, y) \leq T_{1}\left(m_{1}, m_{2}\right)$. Choose $\mathcal{L}_{1}=T_{1}\left(M_{1}, m_{2}\right)$ and $\mathcal{U}_{1}=T_{1}\left(m_{1}, m_{2}\right)$. The proofs for the remaining cases are similar and we skip them to avoid repetition.

The next lemma will be useful later on in this section for showing that a certain sequence of nested invariant attracting rectangular sets cannot intersect in a vertical or a horizontal line. They must either intersect in a point or in a limiting rectangular set.

Lemma 9 Suppose $B_{i} \gamma_{i}-C_{i} \beta_{i} \neq 0$ for $i=1,2$. Consider the system of equations

$$
m_{1}=\mathcal{L}_{1}, \quad M_{1}=\mathcal{U}_{1}, \quad m_{2}=\mathcal{L}_{2}, \quad M_{2}=\mathcal{U}_{2},
$$

where $\mathcal{L}_{1}, \mathcal{U}_{1}, \mathcal{L}_{2}$ and $\mathcal{U}_{2}$ are given by Table 5 of Lemma 8 . Then $m_{1}=M_{1}$ if and only if $m_{2}=M_{2}$.

Proof Suppose $m_{1}=M_{1}$ in (25). Using the formulas for $\mathcal{L}_{2}$ and $\mathcal{U}_{2}$ given in cases 1 and 4 of Table 5, one gets upon subtracting and eliminating the denominators in (25), then subtracting the numerators that $m_{2}=M_{2}$. In case 2 of Table 5 , clearly, $m_{2}=M_{2}$ is a solution of (25). If $m_{2} \neq M_{2}$, then subtracting and eliminating the denominators in (25) gives that $m_{2}$ and $M_{2}$ are solutions of the quadratic

$$
C_{2} t^{2}+\left(A_{2}+B_{2} M_{1}-\gamma_{2}\right) t-\alpha_{2}-M_{1} \beta_{2}
$$

whose roots have opposite signs, giving a contradiction. Moreover, under the assumption $m_{1}=M_{1}$, cases 6,5 and 3 of Table 5 reduce to cases 1,4 and 2, respectively, for $i=2$. The proof for $m_{2}=M_{2}$ is similar and we skip it. 
Table 6 Positions of $K_{i}$ and $L_{i}$ in the set $\mathcal{B}$ for various parameter regions

\begin{tabular}{|c|c|c|c|c|c|c|c|}
\hline Case & $\begin{array}{l}B_{i} \gamma_{i}-C_{i} \beta_{i} \\
i=1,2\end{array}$ & $\begin{array}{l}B_{i} \alpha_{i}-A_{i} \beta_{i} \\
i=1,2\end{array}$ & $\begin{array}{l}C_{i} \alpha_{i}-A_{i} \gamma_{i} \\
i=1,2\end{array}$ & Slope of $E_{1}$ & Slope of $E_{2}$ & The set $\mathcal{B}$ & \\
\hline (i) & $=0$ & $>0$ & $>0$ & - & + & \multicolumn{2}{|c|}{$f_{i}(\downarrow, \downarrow)$} \\
\hline (ii) & $=0$ & $<0$ & $<0$ & + & - & \multicolumn{2}{|c|}{$f_{i}(\uparrow, \uparrow)$} \\
\hline (iii) & $>0$ & $\geq 0$ & $\geq 0$ & + or - & - & $f_{i}(\downarrow, \downarrow)$ & $f_{i}(\downarrow, \uparrow)$ \\
\hline & & & & & & \multicolumn{2}{|c|}{$K_{i}$} \\
\hline (iv) & $>0$ & $\geq 0$ & $<0$ & + & - & \multicolumn{2}{|c|}{$f_{i}(\downarrow, \uparrow)$} \\
\hline \multirow{2}{*}{ (v) } & \multirow{2}{*}{$>0$} & \multirow{2}{*}{$<0$} & \multirow{2}{*}{$<0$} & \multirow{2}{*}{+} & \multirow{2}{*}{+ or - } & \multicolumn{2}{|c|}{$f_{i}(\downarrow, \uparrow)$} \\
\hline & & & & & & \multicolumn{2}{|c|}{$f_{i}(\uparrow, \uparrow)$} \\
\hline \multirow{2}{*}{ (vi) } & \multirow{2}{*}{$<0$} & \multirow{2}{*}{$\geq 0$} & \multirow{2}{*}{$\geq 0$} & \multirow{2}{*}{-} & \multirow{2}{*}{+ or -} & \multicolumn{2}{|c|}{$f_{i}(\uparrow, \downarrow)$} \\
\hline & & & & & & \multicolumn{2}{|c|}{$f_{i}(\downarrow, \downarrow)$} \\
\hline (vii) & $<0$ & $<0$ & $\geq 0$ & - & + & \multicolumn{2}{|c|}{$f_{i}(\uparrow, \downarrow)$} \\
\hline \multirow[t]{2}{*}{ (viii) } & $<0$ & $<0$ & $<0$ & + or - & + & $f_{i}(\uparrow, \uparrow)$ & $f_{i}(\uparrow, \downarrow)$ \\
\hline & & & & & & \multicolumn{2}{|c|}{$K_{i}$} \\
\hline
\end{tabular}

Proof of Theorem 8 We consider five separate cases based on the relative positions of the lines $x=K_{1}, x=K_{2}, y=L_{1}$ and $y=L_{2}$ in the invariant attracting box $\mathcal{B}$. We saw in Section 5 that these lines determine regions of coordinatewise monotonicity for the map $T(x, y)$. Also, note that by Lemma $3, K_{1}$ and $L_{1}$ cannot lie in $[0, \infty)^{2}$ at the same time. Similarly, $K_{2}$ and $L_{2}$ cannot lie in $[0, \infty)^{2}$ at the same time. The five cases are as follows.

(a) $\left\{K_{1}, K_{2}\right\} \cap\left[\mathcal{L}_{1}, \mathcal{U}_{1}\right]=\phi$ and $\left\{L_{1}, L_{2}\right\} \cap\left[\mathcal{L}_{2}, \mathcal{U}_{2}\right]=\phi$.

(b) Either $K_{2} \in\left[\mathcal{L}_{1}, \mathcal{U}_{1}\right]$ or $L_{1} \in\left[\mathcal{L}_{2}, \mathcal{U}_{2}\right]$, and $K_{1} \notin\left[\mathcal{L}_{1}, \mathcal{U}_{1}\right], L_{2} \notin\left[\mathcal{L}_{2}, \mathcal{U}_{2}\right]$.

(c) Either $K_{1} \in\left[\mathcal{L}_{1}, \mathcal{U}_{1}\right]$ or $L_{2} \in\left[\mathcal{L}_{2}, \mathcal{U}_{2}\right]$, and $K_{2} \notin\left[\mathcal{L}_{1}, \mathcal{U}_{1}\right], L_{1} \notin\left[\mathcal{L}_{2}, \mathcal{U}_{2}\right]$.

(d) $K_{2}, L_{1} \in\left[\mathcal{L}_{1}, \mathcal{U}_{1}\right]$ or $K_{1}, L_{2} \in\left[\mathcal{L}_{2}, \mathcal{U}_{2}\right]$.

(e) $K_{1}, K_{2} \in\left[\mathcal{L}_{1}, \mathcal{U}_{1}\right]$ or $L_{1}, L_{2} \in\left[\mathcal{L}_{2}, \mathcal{U}_{2}\right]$.

Case (a): A direct inspection of Table 6 shows that there are four regions of coordinatewise monotonicities of system (1) which satisfy case (a), namely,

\begin{tabular}{|l|l|}
\hline$f_{1}(\uparrow, \downarrow)$ \\
$f_{2}(\downarrow, \uparrow)$
\end{tabular}$\quad \begin{aligned} & f_{1}(\downarrow, \uparrow) \\
& f_{2}(\uparrow, \downarrow)\end{aligned} \quad \begin{aligned} & f_{1}(\uparrow, \downarrow) \\
& f_{2}(\uparrow, \downarrow)\end{aligned} \quad$\begin{tabular}{l}
$f_{1}(\downarrow, \uparrow)$ \\
$f_{2}(\downarrow, \uparrow)$ \\
\hline
\end{tabular}


Figure 9 The dots indicate corners of a nested sequence of boxes in case (c).

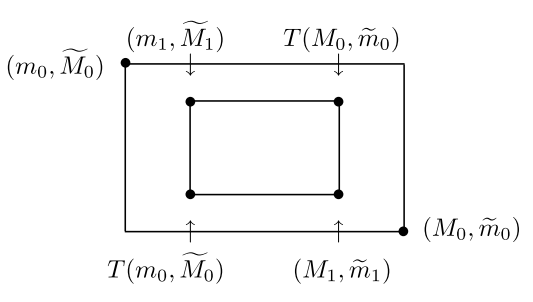

In the first case, the map $T$ is competitive and in the second case, the map $T^{2}$ is competitive. Hence one can use the theory of competitive maps in [33] and the methodology in [3] to show that every orbit converges to one of three equilibria or to the unique minimal period-two solution discussed in Section 8.3. In the third case, we consider a specific example of a parameter region from Table 6 which corresponds to this type of monotonicity, namely,

$$
\begin{aligned}
& B_{1} \gamma_{1}-C_{1} \beta_{1}<0, \quad B_{1} \alpha_{1}-A_{1} \beta_{1}<0, \quad C_{1} \alpha_{1}-A_{1} \gamma_{1} \geq 0, \\
& B_{2} \gamma_{2}-C_{2} \beta_{2}<0, \quad B_{2} \alpha_{2}-A_{2} \beta_{2}<0, \quad C_{2} \alpha_{2}-A_{2} \gamma_{2} \geq 0 \text {. }
\end{aligned}
$$

In this case, the coordinatewise monotonicities of $T_{1}(x, y)$ and $T_{2}(x, y)$ give

$$
\left(x_{1}, y_{1}\right) \preceq_{\text {se }}\left(x_{2}, y_{2}\right) \quad \Longrightarrow \quad T\left(x_{1}, y_{1}\right)<T\left(x_{2}, y_{2}\right) .
$$

Set $m_{0}:=\mathcal{L}_{1}, M_{0}:=\mathcal{U}_{1}, \widetilde{m}_{0}:=\mathcal{L}_{2}$ and $\widetilde{M}_{0}:=\mathcal{U}_{2}$, where $\mathcal{L}_{1}, \mathcal{U}_{1}, \mathcal{L}_{2}$ and $\mathcal{U}_{2}$ are as given in Table 5 of Lemma 8 . For $n=0,1,2, \ldots$, define

$$
\begin{array}{ll}
m_{n+1}=T_{1}\left(m_{n}, \tilde{M}_{n}\right), & M_{n+1}=T_{1}\left(M_{n}, \widetilde{m}_{n}\right), \\
\tilde{m}_{n+1}=T_{2}\left(m_{n}, \tilde{M}_{n}\right), & \widetilde{M}_{n+1}=T_{2}\left(M_{n}, \widetilde{m}_{n}\right)
\end{array}
$$

as shown in Figure 9.

Since the slopes of the equilibrium curves $E_{1}$ and $E_{2}$ have opposite signs in the invariant attracting set $\left[\mathcal{L}_{1}, \mathcal{U}_{1}\right] \times\left[\mathcal{L}_{2}, \mathcal{U}_{2}\right]$, the curves must intersect exactly once there. Thus system $(1)$ must have a unique equilibrium in $\left[\mathcal{L}_{1}, \mathcal{U}_{1}\right] \times\left[\mathcal{L}_{2}, \mathcal{U}_{2}\right]$ which must be an interior equilibrium. As a result, $\left(\mathcal{L}_{1}, \mathcal{U}_{2}\right)$ and $\left(\mathcal{U}_{1}, \mathcal{L}_{2}\right)$ cannot be fixed points of the map $T$. This and (26) imply that for $(x, y) \in\left[\mathcal{L}_{1}, \mathcal{U}_{1}\right] \times\left[\mathcal{L}_{2}, \mathcal{U}_{2}\right]$,

$$
\left(m_{0}, \widetilde{M}_{0}\right) \preceq_{\text {se }} T\left(m_{0}, \tilde{M}_{0}\right)<T(x, y)<T\left(M_{0}, \widetilde{m}_{0}\right) \preceq_{\text {se }}\left(M_{0}, \widetilde{m}_{0}\right) .
$$

Equations (27) and (28) give: $\left(m_{0}, \widetilde{M}_{0}\right) \preceq_{\text {se }}\left(m_{1}, \widetilde{M}_{1}\right) \preceq_{\text {se }} T(x, y) \preceq_{\text {se }}\left(M_{1}, \widetilde{m}_{1}\right) \preceq_{\text {se }}\left(M_{0}, \widetilde{m}_{0}\right)$. Hence, by (26), $T\left(m_{0}, \widetilde{M}_{0}\right)<T\left(m_{1}, \widetilde{M}_{1}\right)<T^{2}(x, y)<T\left(M_{1}, \widetilde{m}_{1}\right)<T\left(M_{0}, \widetilde{m}_{0}\right)$.

Continuing in this manner, one has for $(x, y) \in\left[\mathcal{L}_{1}, \mathcal{U}_{1}\right] \times\left[\mathcal{L}_{2}, \mathcal{U}_{2}\right]$,

$$
\begin{aligned}
T\left(m_{n}, \tilde{M}_{n}\right) & <T\left(m_{n+1}, \widetilde{M}_{n+1}\right)<T^{n+2}(x, y)<T\left(M_{n+1}, \widetilde{m}_{n+1}\right) \\
& <T\left(M_{n}, \widetilde{m}_{n}\right), \quad n=0,1,2, \ldots
\end{aligned}
$$

Since $T\left(m_{n}, \widetilde{M}_{n}\right)<(\bar{x}, \bar{y})<T\left(M_{n}, \widetilde{m}_{n}\right)$ for all integers $n \geq 0$, it follows from (29) that $\lim _{n \rightarrow \infty} T^{n+2}(x, y)=(\bar{x}, \bar{y})$. Thus every solution of system (1) converges to the unique equilibrium $(\bar{x}, \bar{y})$. In the last case, the coordinatewise monotonicities of $T_{1}(x, y)$ and $T_{2}(x, y)$ 
Figure 10 The dots indicate corners of a nested sequence of boxes in case (b).

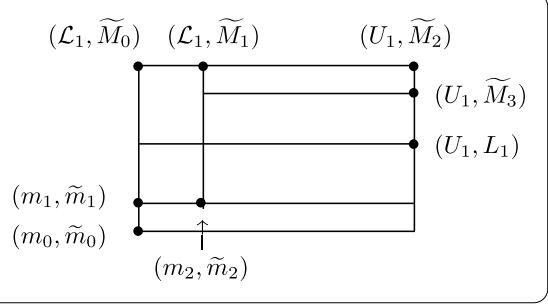

give $\left(x_{1}, y_{1}\right) \preceq_{\text {se }}\left(x_{2}, y_{2}\right)$, which implies $T\left(x_{2}, y_{2}\right)<T\left(x_{1}, y_{1}\right)$. The rest of the proof is similar to the proof of the previous case and we skip it to avoid repetition.

Case (b) Example 1: Once again we give the proof for a specific example of a parameter region from Table 6 which corresponds to this type of monotonicity, namely,

$$
\begin{aligned}
& B_{1} \gamma_{1}-C_{1} \beta_{1}>0, \quad B_{1} \alpha_{1}-A_{1} \beta_{1}<0, \quad C_{1} \alpha_{1}-A_{1} \gamma_{1}<0, \\
& B_{2} \gamma_{2}-C_{2} \beta_{2}>0, \quad B_{2} \alpha_{2}-A_{2} \beta_{2} \geq 0, \quad C_{2} \alpha_{2}-A_{2} \gamma_{2}<0 .
\end{aligned}
$$

In this case, the horizontal line $y=L_{1} \subset \mathcal{B}$. Table 5 of Lemma 8 gives that the equilibrium curve $E_{2}$ is decreasing for the parameter region in case (a). Moreover, in this case, $T_{2}(x, y)$ is nonincreasing in $x$ and nondecreasing in $y$ on $\left[\mathcal{L}_{1}, \mathcal{U}_{1}\right] \times\left[\mathcal{L}_{2}, \mathcal{U}_{2}\right]$ and hence

$$
\min _{(x, y) \in\left[\mathcal{L}_{1}, \mathcal{U}_{1}\right] \times\left[\mathcal{L}_{2}, \mathcal{U}_{2}\right]} T_{2}(x, y)=\lim _{\substack{x \rightarrow \infty \\ y \rightarrow 0}} \frac{\alpha_{2}+\beta_{2} x+\gamma_{2} y}{A_{2}+B_{2} x+C_{2} y}=\frac{\beta_{2}}{B_{2}} .
$$

Set $m_{0}:=m_{1}:=\mathcal{L}_{1}, \widetilde{m}_{0}:=\frac{\beta_{2}}{B_{2}}$ and $\widetilde{M}_{0}:=\widetilde{M}_{1}:=\widetilde{M}_{2}:=\mathcal{U}_{2}$. For $n=0,1,2, \ldots$, define

$$
m_{n+2}:=T_{1}\left(m_{n+1}, \widetilde{m}_{n+1}\right), \quad \widetilde{m}_{n+1}:=T_{2}\left(\mathcal{U}_{1}, \widetilde{m}_{n}\right), \quad \widetilde{M}_{n+3}:=T_{2}\left(m_{2}, \widetilde{M}_{n+2}\right)
$$

as given in Figure 10.

Note that $y=\frac{\beta_{2}}{B_{2}}$ is a horizontal asymptote of $E_{2}:=\left\{(x, y) \mid y=T_{2}(x, y)\right\}$ and hence the point $\left(\mathcal{U}_{1}, \widetilde{m}_{0}\right)$ lies in the region below $E_{2}$. Since the point $(0,0)$, which also lies in this region, satisfies $0<T_{2}(0,0)$, one must have $\widetilde{m}_{0}<T_{2}\left(\mathcal{U}_{1}, \widetilde{m}_{0}\right)=\widetilde{m}_{1}$. Also note that $T_{1}(x, y)$ is nondecreasing in both $x$ and $y$ on $\left[\mathcal{L}_{1}, \mathcal{U}_{1}\right] \times\left[\mathcal{L}_{2}, L_{1}\right]$, and it is nonincreasing in $x$ and nondecreasing in $y$ on $\left[\mathcal{L}_{1}, \mathcal{U}_{1}\right] \times\left[L_{1}, \mathcal{U}_{2}\right]$. This gives

$$
\begin{aligned}
& m_{0} \leq m_{1} \quad \text { and } \quad \widetilde{m}_{0}<\widetilde{m}_{1} \quad \Longrightarrow \quad m_{1}=T_{1}\left(m_{0}, \widetilde{m}_{0}\right)<T_{1}\left(m_{1}, \widetilde{m}_{1}\right)=m_{2} \\
& \Longrightarrow \quad \tilde{M}_{3}=T_{2}\left(m_{2}, \mathcal{U}_{2}\right)<T_{2}\left(m_{1}, \mathcal{U}_{2}\right)=\widetilde{M}_{2} \text {. }
\end{aligned}
$$

Moreover, the coordinatewise monotonicities of $T_{1}(x, y)$ and $T_{2}(x, y)$ imply

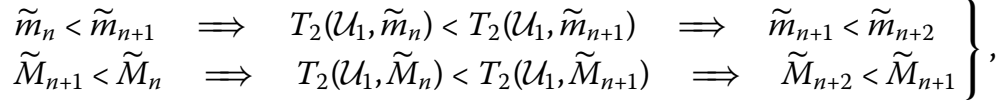

$$
\begin{aligned}
& n=1,2,3, \ldots .
\end{aligned}
$$


From (31) and (32), we have

$$
\begin{aligned}
& \tilde{m}_{n}<\tilde{m}_{n+1}<\bar{y}<\tilde{M}_{n+1}<\tilde{M}_{n}, \quad n=1,2,3, \ldots \\
& \quad \Longrightarrow \quad\left[m_{2}, \mathcal{U}_{1}\right] \times\left[\widetilde{m}_{n+1}, \widetilde{M}_{n+1}\right] \subset\left[m_{2}, \mathcal{U}_{1}\right] \times\left[\widetilde{m}_{n}, \widetilde{M}_{n}\right], \quad n=1,2,3, \ldots
\end{aligned}
$$

So, either $L_{1}<\tilde{m}_{N}$ or $\tilde{M}_{N}<L_{1}$ for some $N$, or $L_{1}=\lim \tilde{m}_{n}=\lim \widetilde{M}_{n}=\bar{y}$. In the first two cases, the proof of global convergence to the unique equilibrium $(\bar{x}, \bar{y})$ is similar to case (a) and we skip it. In the last case, recall that $\left.\frac{\partial}{\partial x} T_{1}(x, y)\right|_{y=L_{1}}=0$. Since the equilibrium $(\bar{x}, \bar{y})$ lies on the line $y=L_{1}$, it follows that $\mathcal{L}_{1}=\mathcal{U}_{1}=\bar{x}$ giving global convergence to $(\bar{x}, \bar{y})$.

Case (b) Example 2: In some parameter regions, it is possible to get multiple equilibria or a unique minimal period-two solution but not both (see Proposition 1 in Section 8.3). For example, consider the parameter region

$$
\begin{aligned}
& B_{1} \gamma_{1}-C_{1} \beta_{1}<0, \quad B_{1} \alpha_{1}-A_{1} \beta_{1} \geq 0, \quad C_{1} \alpha_{1}-A_{1} \gamma_{1} \geq 0, \\
& B_{2} \gamma_{2}-C_{2} \beta_{2}>0, \quad B_{2} \alpha_{2}-A_{2} \beta_{2} \geq 0, \quad C_{2} \alpha_{2}-A_{2} \gamma_{2}<0 \text {. }
\end{aligned}
$$

In this case, set $m_{0}:=\mathcal{L}_{1}, \widetilde{m}_{0}:=\mathcal{L}_{2}, M_{0}:=\mathcal{U}_{1}$ and $\widetilde{M}_{0}:=\mathcal{U}_{2}$ and define

$$
\begin{array}{ll}
m_{n+1}:=T_{1}\left(m_{n}, \tilde{M}_{n}\right), & M_{n+1}:=T_{1}\left(M_{n}, \tilde{m}_{n}\right), \\
\tilde{m}_{n+1}:=T_{2}\left(M_{n}, \tilde{m}_{n}\right), & \widetilde{M}_{n+1}:=T_{2}\left(m_{n}, \widetilde{M}_{n}\right) .
\end{array}
$$

Let $\lim _{n \rightarrow \infty} m_{n}=m_{1}^{*}, \lim _{n \rightarrow \infty} M_{n}=M_{1}^{*}, \lim _{n \rightarrow \infty} \tilde{m}_{n}=m_{2}^{*}$ and $\lim _{n \rightarrow \infty} \tilde{M}_{n}=M_{2}^{*}$. Consider the equations

$$
\left\{\begin{array}{l}
T_{1}\left(m_{1}^{*}, M_{2}^{*}\right)=m_{1}^{*}, \\
T_{1}\left(m_{1}^{*}, m_{2}^{*}\right)=M_{1}^{*}, \\
T_{2}\left(M_{1}^{*}, m_{2}^{*}\right)=m_{2}^{*}, \\
T_{2}\left(m_{1}^{*}, M_{2}^{*}\right)=M_{2}^{*} .
\end{array}\right.
$$

From (34), we have

$$
\begin{aligned}
& T\left(m_{1}^{*}, M_{2}^{*}\right)=\left(T_{1}\left(m_{1}^{*}, M_{2}^{*}\right), T_{2}\left(m_{1}^{*}, M_{2}^{*}\right)\right)=\left(m_{1}^{*}, M_{2}^{*}\right), \\
& T\left(M_{1}^{*}, m_{2}^{*}\right)=\left(T_{1}\left(M_{1}^{*}, m_{2}^{*}\right), T_{2}\left(M_{1}^{*}, m_{2}^{*}\right)\right)=\left(M_{1}^{*}, m_{2}^{*}\right) .
\end{aligned}
$$

Thus $\left(m_{1}^{*}, M_{2}^{*}\right)$ and $\left(M_{1}^{*}, m_{2}^{*}\right)$ are additional equilibria of system (1) alongside $(\bar{x}, \bar{y})$. The three equilibria are ordered by the south-east partial ordering $\preceq_{\text {se }}$ as follows:

$$
\left(m_{1}^{*}, M_{2}^{*}\right) \preceq_{\mathrm{se}}(\bar{x}, \bar{y}) \preceq_{\mathrm{se}}\left(M_{1}^{*}, m_{2}^{*}\right) .
$$

Note that in this case, $L_{1} \in\left[m_{1}^{*}, M_{1}^{*}\right] \times\left[m_{2}^{*}, M_{2}^{*}\right]$. We consider two possibilities for the $y$-coordinate of $(\bar{x}, \bar{y})$ : (i) $\bar{y} \geq L_{1}$ and (ii) $\bar{y}<L_{1}$. In the first case, one can use methods introduced earlier in the proof to show that the points in the region $\left[m_{1}^{*}, \bar{x}\right] \times\left[m_{2}^{*}, L_{2}\right]$ upon repeated iteration enter the region above the line $y=L_{1}$, that is, the region $\left[m_{1}^{*}, M_{1}^{*}\right] \times$ $\left[L_{1}, M_{2}^{*}\right]$. Note that the map $T(x, y)$ is competitive in this region. It follows from the theory of competitive maps (see [33]) that every solution converges to an equilibrium. 
Figure 11 Regions of coordinatewise monotonicity when (left) both $K_{2}$ and $L_{1}$ lie in the invariant attracting set $\mathcal{B}$ and (right) both $K_{1}$ and $K_{2}$ lie in $\mathcal{B}$.
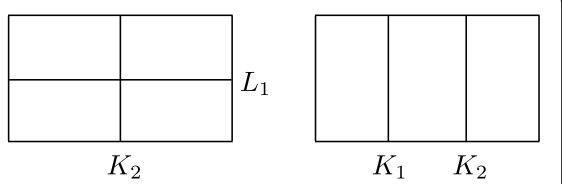

Next we show that the case $\bar{y}<L_{1}$ cannot exist. Suppose, for the sake of contradiction, that it did. In particular, look at the region $\left[m_{1}^{*}, \bar{x}\right] \times\left[m_{2}^{*}, \bar{y}\right]$. Since the first coordinate of the map $T(x, y):=\left(T_{1}(x, y), T_{2}(x, y)\right)$ satisfies $\frac{\partial T_{1}}{\partial x}<0, \frac{\partial T_{1}}{\partial y}<0$, one must have

$$
\left(m_{1}^{*}, m_{2}^{*}\right) \leq(x, y) \leq(\bar{x}, \bar{y}) \quad \Longrightarrow \quad \bar{x}=T_{1}(\bar{x}, \bar{y}) \leq T_{1}(x, y) \leq T_{1}\left(m_{1}^{*}, m_{2}^{*}\right)
$$

Thus $T\left(\left[m_{1}^{*}, \bar{x}\right] \times\left[m_{2}^{*}, \bar{y}\right]\right) \subset\left[\bar{x}, M_{1}^{*}\right] \times\left[m_{2}^{*}, M_{2}^{*}\right]$. Similarly, one can show that $T\left(\left[\bar{x}, M_{1}^{*}\right] \times\right.$ $\left.\left[m_{2}^{*}, \bar{y}\right]\right) \subset\left(\left[m_{1}^{*}, \bar{x}\right] \times\left[m_{2}^{*}, M_{2}^{*}\right]\right)$. This indicates that solutions spiral about the saddle equilibrium $(\bar{x}, \bar{y})$, which implies that the Jacobian of $T(\bar{x}, \bar{y})$ must have complex eigenvalues. But we know that the eigenvalues of the latter satisfy $\left|\lambda_{1}\right|<1$ and $\left|\lambda_{2}\right|>1$. Hence they must be real by Theorem 10, giving a contradiction.

The proof for the case where minimal period-two solutions exist is similar with the map $T(x, y)$ replaced by $T^{2}(x, y)$. The proof of case (c) is similar to that of case (b) and we skip it. In cases $(\mathrm{d})$ and (e), the regions of coordinatewise monotonicity are as shown in Figure 11. It is straightforward to verify that in these two cases, repeated iteration of the map $T(x, y)$ causes the shrinking sequence of invariant attracting boxes $\left\{\left[m_{n}, M_{n}\right] \times\left[\tilde{m}_{n}, \tilde{M}_{n}\right]\right\}_{n=0}^{\infty}$ to enter one of the regions given in cases (a), (b) and (c). The proof is a direct consequence of this.

\section{Appendix: Theorems on global dynamics of system (1) when both equilibrium curves are reducible conics}

Theorem 14 If the graphs of $E_{1}$ and $E_{2}$ are each pairs of perpendicular lines

$$
\begin{aligned}
& E_{1}=\left\{(x, y) \in \mathbb{R}^{2}: x\left(C_{1} y+A_{1}-\beta_{1}\right)=0\right\}, \\
& E_{2}=\left\{(x, y) \in \mathbb{R}^{2}: y\left(B_{2} x+A_{2}-\gamma_{2}\right)=0\right\},
\end{aligned}
$$

then system (1) must have infinitely many equilibria or at most two finite equilibria in $[0, \infty)^{2}$, namely $\mathcal{E}_{0}$ and $\mathcal{E}_{3} . \mathcal{E}_{0}$ is always an equilibrium. In addition, there exist unbounded solutions. The equilibria and their basins of attraction must satisfy Table 7.

Theorem 15 If the graphs of $E_{1}$ and $E_{2}$ are respectively pairs of parallel and transversal lines with formulas

$$
\begin{aligned}
& E_{1}=\left\{(x, y) \in \mathbb{R}^{2}: x^{2}+\left(A_{1}-\beta_{1}\right) x-\alpha_{1}=0\right\}, \\
& E_{2}=\left\{(x, y) \in \mathbb{R}^{2}: C_{2} y^{2}+B_{2} x y+\left(A_{2}-\gamma_{2}\right) y-\beta_{2} x-\alpha_{2}=0\right\},
\end{aligned}
$$

then $\mathcal{E}_{0}$ is always an equilibrium of system (1). In addition,

(a) If $\beta_{1}-A_{1}<0$ and $\gamma_{2}-A_{2} \leq 0$, then the unique equilibrium $\mathcal{E}_{0}$ is globally asymptotically stable.

(b) If $\beta_{1}-A_{1}=0$, then there exist infinitely many equilibria along the nonnegative $x$-axis. 
Table 7 Global behavior of solutions when $E_{1}$ and $E_{2}$ are pairs of perpendicular lines

\begin{tabular}{llll}
\hline Parameter region & $\mathcal{E}_{\mathbf{0}}$ & $\mathcal{E}_{\mathbf{3}}$ & Global dynamics \\
\hline$\beta_{1}-A_{1}<0$ & G.A.S. & - & Every soln. converges to $\mathcal{E}_{0}$. \\
$\gamma_{2}-A_{2}<0$ & $\begin{array}{l}\text { Basin of attraction: } \\
{[0, \infty)^{2}}\end{array}$ & & \\
$\beta_{1}-A_{1}<0$ & Repeller & - & Every soln. except $(0,0)$ tends \\
$\gamma_{2}-A_{2}>0$ & & & to $(0, \infty)$. \\
$\beta_{1}-A_{1}>0$ & Repeller & - & Every soln. except $(0,0)$ tends \\
$\gamma_{2}-A_{2}<0$ & & & to $(\infty, 0)$. \\
$\beta_{1}-A_{1}>0$ & Repeller & Saddle & Every soln. not on $\mathcal{C}$ except $(0,0)$ \\
$\gamma_{2}-A_{2}>0$ & & Stable manifold: & tends to $(0, \infty)$ or $(\infty, 0)$. \\
$\beta_{1}-A_{1}=0$, or & & An increasing curve $\mathcal{C}$ & \\
$\gamma_{2}-A_{2}=0$ & & & There exist infinitely many \\
\hline
\end{tabular}

Table 8 Global behavior of solutions when $\beta_{1}-A_{1}>0$ and $\gamma_{2}-A_{2}>0$ when $E_{1}$ and $E_{2}$ are respectively pairs of parallel and transversal lines

\begin{tabular}{lllll}
\hline Parameter region & $\mathcal{E}_{\mathbf{0}}$ & $\mathcal{E}_{\mathbf{1}}$ & $\mathcal{E}_{\mathbf{2}}$ & $\mathcal{E}_{\mathbf{3}}$ \\
\hline$B_{2}\left(\beta_{1}-A_{1}\right) \geq B_{1}\left(\gamma_{2}-A_{2}\right)$ & Repeller & L.A.S. & Saddle & - \\
& & $\begin{array}{l}\text { Basin of attraction: } \\
(0, \infty)^{2} \text { and }\end{array}$ & $\begin{array}{l}\text { Its stable manifold: } \\
\text { positive } x \text {-axis }\end{array}$ & Positive y-axis \\
& & Saddle & Saddle & \\
$B_{2}\left(\beta_{1}-A_{1}\right)<B_{1}\left(\gamma_{2}-A_{2}\right)$ & Repeller & Sa.S. \\
& & Its stable manifold: & Its stable manifold: & Basin of attraction: \\
& & Positive x-axis & Positive $y$-axis & $(0, \infty)^{2}$ \\
\hline
\end{tabular}

(c) If $\beta_{1}-A_{1}>0$ and $\gamma_{2}-A_{2} \leq 0$, then there exist two equilibria, namely $\mathcal{E}_{0}$ and $\mathcal{E}_{1} . \mathcal{E}_{0}$ is a saddle point with the positive $y$-axis as its stable manifold. $\mathcal{E}_{1}$ is LAS and attracts all solutions with initial conditions in $(0, \infty)^{2}$ or on the positive $x$-axis.

(d) If $\beta_{1}-A_{1}>0$ and $\gamma_{2}-A_{2}>0$, then the nonnegative equilibria of system (1) must satisfy Table 8 .

Theorem 16 If the graphs of $E_{1}$ and $E_{2}$ are respectively pairs of perpendicular and transversal lines with formulas

$$
\begin{aligned}
& E_{1}=\left\{(x, y) \in \mathbb{R}^{2}: x\left(C_{1} y+A_{1}-\beta_{1}\right)=0\right\}, \\
& E_{2}=\left\{(x, y) \in \mathbb{R}^{2}: C_{2} y^{2}+B_{2} x y+\left(A_{2}-\gamma_{2}\right) y-\beta_{2} x-\alpha_{2}=0\right\},
\end{aligned}
$$

then the nonnegative equilibria of system (1) must satisfy:

(a) If $\beta_{1}-A_{1} \leq 0$ and $\gamma_{2}-A_{2} \leq 0$, then the unique equilibrium $\mathcal{E}_{0}$ is globally asymptotically stable.

(b) If $\beta_{1}-A_{1}=0$ and $\gamma_{2}-A_{2} \leq 0$, then there exist infinitely many equilibria along the nonnegative $x$-axis.

(c) If $\beta_{1}-A_{1}>0$ and $\gamma_{2}-A_{2} \leq 0$, then $\mathcal{E}_{0}$ is a saddle point with the positive $y$-axis as its stable manifold. All solutions with initial conditions in $(0, \infty)^{2}$ or on the positive $x$-axis are unbounded and tend to $(\infty, 0)$.

(d) If $\beta_{1}-A_{1}>0$ and $\gamma_{2}-A_{2}>0$, then the nonnegative equilibria of system (1) must satisfy Table 9. 
Table 9 Global behavior of solutions when $\beta_{1}-A_{1}>0$ and $\gamma_{2}-A_{2}>0$ when $E_{1}$ and $E_{2}$ are respectively pairs of perpendicular and transversal lines

\begin{tabular}{|c|c|c|c|}
\hline Parameter region & $\mathcal{E}_{0}$ & $\mathcal{E}_{2}$ & $\mathcal{E}_{3}$ \\
\hline$\overline{C_{2}}\left(\beta_{1}-A_{1}\right)>C_{1}\left(\gamma_{2}-A_{2}\right)$ & Repeller & $\begin{array}{l}\text { Saddle } \\
\text { Its stable manifold: } \\
\text { Positive } y \text {-axis }\end{array}$ & - \\
\hline$C_{2}\left(\beta_{1}-A_{1}\right)=C_{1}\left(\gamma_{2}-A_{2}\right)$ & Repeller & $\begin{array}{l}\text { Saddle } \\
\text { Its stable manifold: } \\
\text { Positive } y \text {-axis } \\
\text { Basin of attraction: } \\
(0, \infty) \times(b, \infty) \text {, where } \mathcal{E}_{2}=(0, b) \\
\text { Note: Solns. in }(0, \infty) \times(0, b) \longrightarrow(\infty, 0)\end{array}$ & - \\
\hline$C_{2}\left(\beta_{1}-A_{1}\right)<C_{1}\left(\gamma_{2}-A_{2}\right)$ & Repeller & $\begin{array}{l}\text { L.A.S. } \\
\text { Basin of attraction: } \\
\text { Region above an increasing curve } \mathcal{C}\end{array}$ & $\begin{array}{l}\text { Saddle } \\
\text { Its stable manifold: } \\
\text { The increasing curve } \mathcal{C} \\
\text { Note: Solns. in the region } \\
\text { below } \mathcal{C} \rightarrow(\infty, 0)\end{array}$ \\
\hline
\end{tabular}

Table 10 Global behavior of solutions when $\beta_{1}-A_{1}>0$ and $\gamma_{2}-A_{2}>0$ when $E_{1}$ and $E_{2}$ are respectively pairs of perpendicular and parallel lines

\begin{tabular}{llll}
\hline Parameter region & $\mathcal{E}_{\mathbf{0}}$ & $\mathcal{E}_{\mathbf{2}}$ & Global dynamics \\
\hline$C_{2}\left(\beta_{1}-A_{1}\right)<C_{1}\left(\gamma_{2}-A_{2}\right)$ & Repeller & $\begin{array}{l}\text { L.A.S. } \\
\text { Basin of attraction: } \\
(0, \infty)^{2} \text { and } \\
\text { positive } y \text {-axis }\end{array}$ & $\begin{array}{l}\text { Every soln. on the positive } x \text {-axis is } \\
\text { unbounded and tends to }(\infty, 0) .\end{array}$ \\
$C_{2}\left(\beta_{1}-A_{1}\right)>C_{1}\left(\gamma_{2}-A_{2}\right)$ & Repeller & $\begin{array}{l}\text { Saddle } \\
\text { Its stable manifold: }\end{array}$ & Positive $y$-axis \\
$C_{2}\left(\beta_{1}-A_{1}\right)=C_{1}\left(\gamma_{2}-A_{2}\right)$ & & Every soln. in $(0, \infty)^{2}$ or on the $x$-axis is unbounded and \\
& & tends to $(\infty, 0)$ or $(\infty, c), c>0$. \\
& & There exist infinitely many equilibria \\
& & along the horizontal line $y=\frac{\beta_{1}-A_{1}}{C_{1}}$. \\
\hline
\end{tabular}

Theorem 17 If the graphs of $E_{1}$ and $E_{2}$ are respectively pairs of perpendicular and parallel lines with formulas

$$
\begin{aligned}
& E_{1}=\left\{(x, y) \in \mathbb{R}^{2}: x\left(C_{1} y+A_{1}-\beta_{1}\right)=0\right\}, \\
& E_{2}=\left\{(x, y) \in \mathbb{R}^{2}: C_{2} y^{2}+\left(A_{2}-\gamma_{2}\right) y-\alpha_{2}=0\right\},
\end{aligned}
$$

then the nonnegative equilibria of system (1) must satisfy:

(a) If $\beta_{1}-A_{1}<0$ and $\gamma_{2}-A_{2} \leq 0$, then the unique equilibrium $\mathcal{E}_{0}$ is globally asymptotically stable.

(b) If $\beta_{1}-A_{1}=0$ and $\gamma_{2}-A_{2} \leq 0$, then there exist infinitely many equilibria along the nonnegative $x$-axis.

(c) If $\beta_{1}-A_{1}>0$ and $\gamma_{2}-A_{2} \leq 0$, then $\mathcal{E}_{0}$ is a saddle point with the positive $y$-axis as its stable manifold. All solutions with initial conditions in $(0, \infty)^{2}$ or on the positive $x$-axis are unbounded and tend to $(\infty, 0)$.

(d) If $\beta_{1}-A_{1}>0$ and $\gamma_{2}-A_{2}>0$, then the nonnegative equilibria of system (1) must satisfy Table 10 . 
Table 11 Global dynamics for $\beta_{1}-A_{1}>0$ and $\gamma_{2}-A_{2}>0$ when $E_{1}$ and $E_{2}$ are pairs of transversal lines

\begin{tabular}{|c|c|c|c|c|c|}
\hline & Parameter region & $\mathcal{E}_{0}$ & $\mathcal{E}_{1}$ & $\mathcal{E}_{2}$ & $\mathcal{E}_{3}$ \\
\hline (a) & $\begin{array}{l}B_{2}\left(\beta_{1}-A_{1}\right) \leq B_{1}\left(\gamma_{2}-A_{2}\right) \\
C_{2}\left(\beta_{1}-A_{1}\right)<C_{1}\left(\gamma_{2}-A_{2}\right)\end{array}$ & Repeller & $\begin{array}{l}\text { Saddle } \\
\text { Its stable manifold: } \\
\text { Positive } x \text {-axis }\end{array}$ & $\begin{array}{l}\text { L.A.S. } \\
\text { Basin of attraction: } \\
(0, \infty)^{2} \text { and positive } \\
y \text {-axis }\end{array}$ & - \\
\hline (b) & $\begin{array}{l}B_{2}\left(\beta_{1}-A_{1}\right)<B_{1}\left(\gamma_{2}-A_{2}\right) \\
C_{2}\left(\beta_{1}-A_{1}\right)=C_{1}\left(\gamma_{2}-A_{2}\right)\end{array}$ & Repeller & $\begin{array}{l}\text { Saddle } \\
\text { Its stable manifold: } \\
\text { An increasing curve } \mathcal{C} \\
\text { Basin of attraction: } \\
\text { Region below } \mathcal{C}\end{array}$ & $\begin{array}{l}\text { L.A.S. } \\
\text { Basin of attraction: } \\
\text { Region above } \mathcal{C}\end{array}$ & - \\
\hline (c) & $\begin{array}{l}B_{2}\left(\beta_{1}-A_{1}\right) \geq B_{1}\left(\gamma_{2}-A_{2}\right) \\
C_{2}\left(\beta_{1}-A_{1}\right)>C_{1}\left(\gamma_{2}-A_{2}\right)\end{array}$ & Repeller & $\begin{array}{l}\text { L.A.S. } \\
\text { Basin of attraction: } \\
(0, \infty)^{2} \text { and positive } \\
x \text {-axis }\end{array}$ & $\begin{array}{l}\text { Saddle } \\
\text { Its stable manifold: } \\
\text { Positive } y \text {-axis }\end{array}$ & - \\
\hline (d) & $\begin{array}{l}B_{2}\left(\beta_{1}-A_{1}\right)>B_{1}\left(\gamma_{2}-A_{2}\right) \\
C_{2}\left(\beta_{1}-A_{1}\right)=C_{1}\left(\gamma_{2}-A_{2}\right)\end{array}$ & Repeller & $\begin{array}{l}\text { L.A.S. } \\
\text { Basin of attraction: } \\
\text { Region below an } \\
\text { increasing curve } \mathcal{C}\end{array}$ & $\begin{array}{l}\text { Saddle } \\
\text { Its stable manifold: } \\
\text { The increasing curve } \mathcal{C} \\
\text { Basin of attraction: } \\
\text { Region above } \mathcal{C}\end{array}$ & - \\
\hline (e) & $\begin{array}{l}B_{2}\left(\beta_{1}-A_{1}\right)<B_{1}\left(\gamma_{2}-A_{2}\right) \\
C_{2}\left(\beta_{1}-A_{1}\right)>C_{1}\left(\gamma_{2}-A_{2}\right)\end{array}$ & Repeller & $\begin{array}{l}\text { Saddle } \\
\text { Its stable manifold: } \\
\text { Positive } x \text {-axis }\end{array}$ & $\begin{array}{l}\text { Saddle } \\
\text { Its stable manifold: } \\
\text { Positive } y \text {-axis }\end{array}$ & $\begin{array}{l}\text { L.A.S. } \\
\text { Basin of attraction: } \\
(0, \infty)^{2}\end{array}$ \\
\hline (f) & $\begin{array}{l}B_{2}\left(\beta_{1}-A_{1}\right)>B_{1}\left(\gamma_{2}-A_{2}\right) \\
C_{2}\left(\beta_{1}-A_{1}\right)<C_{1}\left(\gamma_{2}-A_{2}\right)\end{array}$ & Repeller & $\begin{array}{l}\text { L.A.S. } \\
\text { Basin of attraction: } \\
\text { Region below an } \\
\text { increasing curve } \mathcal{C}\end{array}$ & $\begin{array}{l}\text { L.A.S. } \\
\text { Basin of attraction: } \\
\text { Region above } \mathcal{C}\end{array}$ & $\begin{array}{l}\text { Saddle } \\
\text { Its stable manifold: } \\
\text { The increasing curve } \mathcal{C}\end{array}$ \\
\hline (g) & $\begin{array}{l}B_{2}\left(\beta_{1}-A_{1}\right)=B_{1}\left(\gamma_{2}-A_{2}\right) \\
C_{2}\left(\beta_{1}-A_{1}\right)=C_{1}\left(\gamma_{2}-A_{2}\right)\end{array}$ & $\rightarrow$ & Infinitely & Many & Equilibria \\
\hline
\end{tabular}

Theorem 18 If the graphs of $E_{1}$ and $E_{2}$ are each pairs of transversal lines with formulas

$$
\begin{aligned}
& E_{1}=\left\{(x, y) \in \mathbb{R}^{2}: x\left(B_{1} x+C_{1} y+A_{1}-\beta_{1}\right)=0\right\}, \\
& E_{2}=\left\{(x, y) \in \mathbb{R}^{2}: y\left(C_{2} y+B_{2} x+A_{2}-\gamma_{2}\right)=0\right\},
\end{aligned}
$$

then $\mathcal{E}_{0}$ is always an equilibrium of system (1). In addition,

(a) If $\beta_{1}-A_{1} \leq 0$ and $\gamma_{2}-A_{2} \leq 0$, then the unique equilibrium $\mathcal{E}_{0}$ is globally asymptotically stable.

(b) If $\beta_{1}-A_{1}>0$ and $\gamma_{2}-A_{2} \leq 0$, then there exist two equilibria, namely $\mathcal{E}_{0}$ and $\mathcal{E}_{1} . \mathcal{E}_{0}$ is a saddle point with the positive $y$-axis as its stable manifold. $\mathcal{E}_{1}$ is LAS and attracts all solutions with initial conditions in $(0, \infty)^{2}$ or on the positive $x$-axis.

(c) If $\beta_{1}-A_{1} \leq 0$ and $\gamma_{2}-A_{2}>0$, then there exist two equilibria, namely $\mathcal{E}_{0}$ and $\mathcal{E}_{2}$. $\mathcal{E}_{0}$ is a saddle point with the positive $x$-axis as its stable manifold. $\mathcal{E}_{2}$ is LAS and attracts all solutions with initial conditions in $(0, \infty)^{2}$ or on the positive $y$-axis.

(d) If $\beta_{1}-A_{1}>0$ and $\gamma_{2}-A_{2}>0$, then the nonnegative equilibria of system (1) and their basins of attraction must satisfy Table 11. 


\section{Acknowledgements}

The author would like to thank Dr. Orlando Merino from the University of Rhode Island for his valuable suggestions. She would also like to thank three anonymous referees for their careful reviews of the paper.

Received: 3 September 2012 Accepted: 3 September 2013 Published: 07 Nov 2013

\section{References}

1. Diblík, J, Halfarová, H: Explicit general solution of planar linear discrete systems with constant coefficients and weak delays. Adv. Differ. Equ. (2013). doi:10.1186/1687-1847-2013-50

2. Diblík, J, Khusainov, D, Šmarda, Z: Construction of the general solution of planar linear discrete systems with constant coefficients and weak delay. Adv. Differ. Equ. 2009, Article ID 784935 (2009). doi:10.1155/2009/784935

3. Basu, S, Merino, O: On the global behavior of solutions to a planar system of difference equations. Commun. Appl. Nonlinear Anal. 16(1), 89-101 (2009)

4. Kulenović, MRS, Nurkanović, M: Global behavior of a two-dimensional competitive system of difference equations with stocking. Math. Comput. Model. 55(7-8), 1998-2011 (2012)

5. Kulenović, MRS, Nurkanović, M: Basins of attraction of an anti-competitive system of difference equations in the plane. Commun. Appl. Nonlinear Anal. 19(2), 41-53 (2012)

6. Kalabusić, S, Kulenović, MRS, Pilav, E: Multiple attractors for a competitive system of rational difference equations in the plane. Abstr. Appl. Anal. 2011, Article ID 295308 (2011)

7. Kalabusić, S, Kulenović, MRS: Dynamics of certain anti-competitive systems of rational difference equations in the plane. J. Differ. Equ. Appl. 17(11), 1599-1615 (2011)

8. Kalabusić, S, Kulenović, MRS, Pilav, E: Dynamics of a two-dimensional system of rational difference equations of Leslie-Gower type. Adv. Differ. Equ. 2011, 29 (2011)

9. Brett, A, Kulenović, MRS, Kalabusić, S: Global attractivity results in partially ordered complete metric spaces. Nonlinear Stud. 18(2), 141-154 (2011)

10. Kulenović, MRS, Merino, O: Invariant manifolds for competitive discrete systems in the plane. Int. J. Bifurc. Chaos Appl. Sci. Eng. 20(8), 2471-2486 (2010)

11. Kalabusić, S, Kulenović, MRS, Pilav, E: Global dynamics of a competitive system of rational difference equations in the plane. Adv. Differ. Equ. 2009, Article ID 132802 (2009)

12. Garić-Demirović, M, Kulenović, MRS, Nurkanović, M: Global behavior of four competitive rational systems of difference equations in the plane. Discrete Dyn. Nat. Soc. 2009, Article ID 153058 (2009)

13. Brett, A, Kulenović, MRS: Basins of attraction of equilibrium points of monotone difference equations. Sarajevo J. Math. 5(18), 211-233 (2009)

14. Brett, A, Kulenović, MRS, Garić-Demirović, M, Nurkanović, M: Global behavior of two competitive rational systems of difference equations in the plane. Commun. Appl. Nonlinear Anal. 16(3), 1-18 (2009)

15. Camouzis, E, Drymonis, E, Ladas, G, Tikjha, W: Patterns of boundedness of the rational system $x_{n+1}=\alpha_{1} /\left(A_{1}+B_{1} x_{n}+C_{1} y_{n}\right)$ and $y_{n+1}=\left(\alpha_{2}+\beta_{2} x_{n}+\gamma_{2} y_{n}\right) /\left(A_{2}+B_{2} x_{n}+C_{2} y_{n}\right)$. J. Differ. Equ. Appl. 18(1), $89-110$ (2012)

16. Camouzis, E, Drymonis, E, Ladas, G: Patterns of boundedness of the rational system $x_{n+1}=\frac{\alpha_{1}+\beta_{1} x_{n}}{A_{1}+B_{1} x_{n}+C_{1} y_{n}}$ and $y_{n+1}=\frac{\alpha_{2}+\beta_{2} x_{n}+\gamma_{2} y_{n}}{A_{2}+B_{2} x_{n}+C_{2} y_{n}}$. Commun. Appl. Nonlinear Anal. 18(1), 1-23 (2011)

17. Camouzis, E, Drymonis, E, Ladas, G: Patterns of boundedness of the rational system $x_{n+1}=\frac{\alpha_{1}+\beta_{1} x_{n}}{A_{1}+C_{1} y_{n}}$ and $y_{n+1}=\frac{\alpha_{2}+\beta_{2} x_{n}+\gamma_{2} y_{n}}{A_{2}+B_{2} x_{n}+C_{2} y_{n}}$. Fasc. Math. 44, 9-18 (2010)

18. Brett, AM, Camouzis, E, Ladas, G, Lynd, CD: On the boundedness character of a rational system. J. Numer. Math. Stoch. 1(1), 1-10 (2009)

19. Camouzis, E, Gilbert, A, Heissan, M, Ladas, G: On the boundedness character of the system $x_{n+1}=\frac{\alpha_{1}+\gamma_{1} y_{n}}{x_{n}}$ and $y_{n+1}=\frac{\alpha_{2}+\beta_{2} x_{n}+\gamma_{2} y_{n}}{A_{2}+x_{n}+y_{n}}$. Commun. Math. Anal. 7(2), 41-50 (2009)

20. Basu, S, Merino, O: Global behavior of solutions to two classes of second-order rational difference equations. Adv. Differ. Equ. 2009, Article ID 128602 (2009)

21. Stević, S, Diblík, J, Iricanin, B, Šmarda, Z: On some solvable difference equations and systems of difference equations. Abstr. Appl. Anal. 2012, Article ID 541761 (2012). doi:10.1155/2012/541761

22. Stević, S, Diblík, J, Iricanin, B, Šmarda, Z: On the difference equation $x_{n}=a_{n} x_{n-k} /\left(b_{n}+c_{n} x_{n-1} \ldots x_{n-k}\right)$. Abstr. Appl. Anal. 2012, Article ID 409237 (2012). doi:10.1155/2012/409237

23. Stević, S, Diblík, J, Iricanin, B, Šmarda, Z: On a third-order system of difference equations with variable coefficients. Abstr. Appl. Anal. 2012, Article ID 508523 (2012). doi:10.1155/2012/508523

24. Stević, S, Diblík, J, Iricanin, B, Šmarda, Z: On a periodic system of difference equations. Abstr. Appl. Anal. 2012, Article ID 258718 (2012). doi:10.1155/2012/258718

25. Camouzis, E, Drymonis, E, Ladas, G: On the global character of the system $x_{n+1}=\frac{a}{x_{n}+y_{n}}$ and $y_{n+1}=\frac{y_{n}}{B x_{n}+y_{n}}$. Commun. Appl. Nonlinear Anal. 16(2), 51-64 (2009)

26. Camouzis, E, Kulenović, MRS, Ladas, G, Merino, O: Rational systems in the plane. J. Differ. Equ. Appl. 15, 303-323 (2009)

27. Camouzis, E, Ladas, G: Global results on rational systems in the plane, part 1. J. Differ. Equ. Appl. 16, 975-1013 (2010)

28. Leonard, WJ, May, R: Nonlinear aspects of competition between species. SIAM J. Appl. Math. 29, 243-275 (1975)

29. de Mottoni, P, Schiaffino, A: Competition systems with periodic coefficients: a geometric approach. J. Math. Biol. 11 319-335 (1981)

30. Smale, S: On the differential equations of species in competition. J. Math. Biol. 3, 5-7 (1976)

31. Liu, P, Elaydi, N: Discrete competitive and cooperative models of Lotka-Volterra type. J. Comput. Anal. Appl. 3(1), 53-73 (2001)

32. Cushing, JM, et al.: Some discrete competition models and the competitive exclusion principle. J. Differ. Equ. Appl. 10(13-15), 1139-1151 (2004)

33. Kulenović, MRS, Merino, O: Competitive-exclusion versus competitive-coexistence for systems in the plane. Discrete Contin. Dyn. Syst., Ser. B 6, 1141-1156 (2006)

34. Hirsch, MW: Systems of differential equations which are competitive or cooperative: I. Limits sets. SIAM J. Math. Anal. 2(13), 167-179 (1982) 
35. Hirsch, M, Smith, H: Monotone dynamical systems. In: Handbook of Differential Equations: Ordinary Differential Equations, vol. II, pp. 239-357. Elsevier, Amsterdam (2005)

36. Smith, HL: Planar competitive and cooperative difference equations. J. Differ. Equ. Appl. 3, 335-357 (1998)

37. Amleh, AM, Camouzis, E, Ladas, G, Radin, M: Patterns of boundedness of a rational system in the plane. J. Differ. Equ. Appl. 16, 1197-1236 (2010)

38. Camouzis, E, Drymonis, E, Ladas, G, Tikjha, W: Patterns of boundedness of the rational system $x_{n+1}=\frac{\alpha_{1}}{A_{1}+B_{1} x_{n}+C_{1} y_{n}}$ and $y_{n+1}=\frac{\alpha_{2}+\beta_{2} x_{n}+\gamma_{2} y_{n}}{A_{2}+B_{2} x_{n}+y_{n}}$. J. Differ. Equ. Appl. 18(7), 1205-1252 (2012)

39. Palladino, FJ: A bifurcation result for a system of two rational difference equations. Adv. Dyn. Syst. Appl. 7(1), 109-128 (2012)

40. Lugo, G, Palladino, FJ: On the boundedness character of rational systems in the plane. J. Differ. Equ. Appl. 17(12) 1801-1811 (2011)

41. Camouzis, E, Ladas, G, Wu, L: On the global character of the system $x_{n+1}=\frac{\alpha_{1}+\gamma_{1} y_{n}}{x_{n}}$ and $y_{n+1}=\frac{\beta_{2} x_{n}+\gamma_{2} y_{n}}{B_{2} x_{n}+C_{2} y_{n}}$. Int. J. Pure Appl. Math. 53(1), 21-36 (2009)

42. Garić-Demirović, M, Kulenović, MRS, Nurkanović, M: Basins of attraction of equilibrium points of second order difference equations. Appl. Math. Lett. 25(12), 2110-2115 (2012)

43. Silverman, JH, Tate, J: Rational Points on Elliptic Curves. Springer, New York (1992)

44. Knapp, AW: Elliptic Curves. Princeton University Press, Princeton (1992)

45. Walker, RJ: Algebraic Curves. Princeton University Press, Princeton (1950)

10.1186/1687-1847-2013-292

Cite this article as: Basu: The roles of conic sections and elliptic curves in the global dynamics of a class of planar systems of rational difference equations. Advances in Difference Equations 2013, 2013:292

\section{Submit your manuscript to a SpringerOpen ${ }^{\circ}$ journal and benefit from:}

- Convenient online submission

- Rigorous peer review

- Immediate publication on acceptance

Open access: articles freely available online

- High visibility within the field

- Retaining the copyright to your article 